\title{
Exact and asymptotic tests for possibly non-regular hypotheses on stochastic volatility models *
}

\author{
Jean-Marie Dufour $^{\dagger} \quad$ Pascale Valéry ${ }^{\ddagger}$ \\ McGill University HEC Montréal
}

First version: March 2002

Revised: June 2004, December 2006

This version: October 7, 2008

Compiled: October 7, 2008, 3:15pm

This paper is forthcoming in the Journal of Econometrics.

\footnotetext{
${ }^{*}$ The authors thank Eric Jacquier, Lynda Khalaf, Nour Meddahi, the Editors Chung-Ming Kuan and Yong-Miao Hong, and two anonymous referees for several helpful comments. This work was supported by the William Dow Chair in Political Economy (McGill University), the Canada Research Chair Program (Chair in Econometrics, Université de Montréal), the Bank of Canada (Research Fellowship), a Guggenheim Fellowship, a Konrad-Adenauer Fellowship (Alexandervon-Humboldt Foundation, Germany), the Institut de finance mathématique de Montréal (IFM2), the Canadian Network of Centres of Excellence [program on Mathematics of Information Technology and Complex Systems (MITACS)], the Natural Sciences and Engineering Research Council of Canada, the Social Sciences and Humanities Research Council of Canada, and the Fonds de recherche sur la société et la culture (Québec).

$\dagger$ William Dow Professor of Economics, McGill University, Centre interuniversitaire de recherche en analyse des organisations (CIRANO), and Centre interuniversitaire de recherche en économie quantitative (CIREQ). Mailing address: Department of Economics, McGill University, Leacock Building, Room 519, 855 Sherbrooke Street West, Montréal, Québec H3A 2T7, Canada. TEL: (1) 514398 8879; FAX: (1) 514398 4938; e-mail: jean-marie.dufour@ mcgill.ca . Web page: http://www.jeanmariedufour.com

¥ Service de l'enseignement de la finance, École des Hautes Études Commerciales de Montréal (HEC-Montréal), 3000 chemin de la Côte-Sainte-Catherine Montréal, Québec), Canada H3T 2A7. TEL: 1 (514) 340-7004, FAX: (514) 340-5632. email: pascale.valery@hec.ca . Web page: http://neumann.hec.ca/pages/pascale.valery/pascalevalery.htm
} 


\begin{abstract}
We study the problem of testing hypotheses on the parameters of one- and two-factor stochastic volatility models (SV), allowing for the possible presence of nonregularities such as singular moment conditions and unidentified parameters, which can lead to non-standard asymptotic distributions. We focus on the development of simulation-based exact procedures - whose level can be controlled in finite samples - as well as on large-sample procedures which remain valid under non-regular conditions. We consider Wald-type, score-type and likelihood-ratio-type tests based on a simple moment estimator, which can be easily simulated. We also propose a $C(\alpha)$-type test which is very easy to implement and exhibits relatively good size and power properties. Besides usual linear restrictions on the SV model coefficients, the problems studied include testing homoskedasticity against a SV alternative (which involves singular moment conditions under the null hypothesis) and testing the null hypothesis of one factor driving the dynamics of the volatility process against two factors (which raises identification difficulties). Three ways of implementing the tests based on alternative statistics are compared: asymptotic critical values (when available), a local Monte Carlo (or parametric bootstrap) test procedure, and a maximized Monte Carlo (MMC) procedure. The size and power properties of the proposed tests are examined in a simulation experiment. The results indicate that the $C(\alpha)$-based tests (built upon the simple moment estimator available in closed form) have good size and power properties for regular hypotheses, while Monte Carlo tests are much more reliable than those based on asymptotic critical values. Further, in cases where the parametric bootstrap appears to fail (for example, in the presence of identification problems), the MMC procedure easily controls the level of the tests. Moreover, MMC-based tests exhibit relatively good power performance despite the conservative feature of the procedure. Finally, we present an application to a time series of returns on the Standard and Poor's Composite Price Index.
\end{abstract}

Key words: testing; exact test; Monte Carlo test; maximized Monte Carlo test; Wald test; LR test; LM test; $C(\alpha)$ test; homoskedasticity; stochastic volatility; two-factor volatility; identification; singular moment conditions; finance; stock prices.

JEL classification: C1, C12, C13, C15, C32, G1. 


\section{RÉSUMÉ}

Dans ce texte, nous étudions des tests d'hypothèses sur les paramètres de modèles de volatilité stochastique (SV) à un ou deux facteurs, en permettant la présence de non-régularités, tels que la singularité locale des conditions de moment définissant l'estimateur ou encore des paramètres de nuisance non-identifiés, ce qui peut conduire à une théorie distributionnelle non standard. Nous développons des procédures exactes dont la taille peut être contrôlée pour une taille donnée d'échantillon, ainsi que des tests justifiés par des arguments asymptotiques, lesquels sont à la fois simples du point de vue numérique et relativement fiables sur de petits échantillons. Nous considérons des critères de types Wald, score et quotient de vraisemblance fondés sur un estimateur des moments (et non sur le maximum de vraisemblance) qui est simple du point de vue numérique. Nous proposons aussi un test de type $C(\alpha)$ qui est très facile à utiliser et qui affiche de bonnes propriétés de niveau et de puissance. Outre des tests de restrictions linéaires sur les coefficients du modèle de volatilité stochastique, les problèmes étudiés comprennent des tests d'homoscédasticité (contre un modèle de volatilité stochastique) et des tests de l'hypothèse nulle d'une volatilité à un facteur contre une volatilité à deux facteurs, lesquels soulèvent des problèmes de singularité locale et d'identification. Nous comparons trois variantes différentes de chaque test suivant que l'on utilise des points critiques asymptotiques standards, une procédure de test de Monte Carlo (ou bootstrap paramétrique) et une procédure de test de Monte Carlo maximisé (MMC). Le niveau et la puissance des procédures proposées sont étudiées par simulation. Les résultats soulignent la supériorité du test $C(\alpha)$ dans les cas réguliers, à la fois pour le niveau et la puissance, tandis que les tests de Monte Carlo s'avèrent plus fiables que leurs homologues asymptotiques. En outre, dans des situations où le bootstrap paramétrique ne parvient pas à contrôler le niveau (par exemple, en présence de problèmes d'identification), la procédure MMC contrôle facilement le niveau des tests. De plus, les tests fondés sur la procédure MMC affichent une bonne puissance bien que cette méthode soit conservatrice par construction. Finalement, nous présentons une application à une série de rendements quotidiens de l'indice boursier du Standard and Poor's.

Mots clé: test d'hypothèse; test exact; tests de Monte Carlo; test de Monte Carlo maximisé; test de Wald; test du score; test du quotient de vraisemblance; test $C(\alpha)$; volatilité stochastique; volatilité à deux facteurs; identification; conditions de moments singulières; finance; prix d'actions.

\section{JEL classification: C1, C12, C13, C15, C32, G1.}




\section{Contents}

1. Introduction 1

2. Framework 4

2.1. One-factor SV model . . . . . . . . . . . . . . . . . . . . . . . 4

2.2. Two-factor SV model . . . . . . . . . . . . . . . . . . . . . 7

3. Test statistics and confidence sets 9

$\begin{array}{lr}\text { 4. Non-regular problems } & 12\end{array}$

$\begin{array}{lll}\text { 5. Monte Carlo tests } & 14\end{array}$

$\begin{array}{ll}\text { 6. Simulation results } & 17\end{array}$

6.1. Level . . . . . . . . . . . . . . . . . . . . . . . . 19

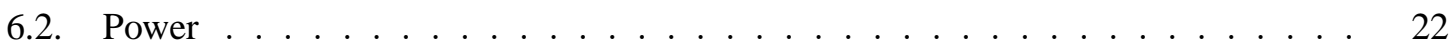

7. Empirical application $\quad 23$

7.1. Data . . . . . . . . . . . . . . . . . . 23

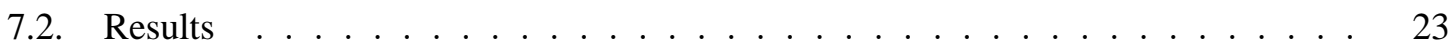

$\begin{array}{llr}\text { 8. Conclusion } & 24\end{array}$

$\begin{array}{ll}\text { A. Appendix: Proofs } & 27\end{array}$

B. Appendix: Analytical moment derivatives for one-factor SV 28

C. Appendix: Analytical moment derivatives for two-factor SV 28 


\section{List of Propositions and Theorems}

2.4 Proposition : Moments of the two-factor SV process . . . . . . . . . . . 8

Proof of Proposition $2.4 \ldots \ldots \ldots \ldots$

\section{List of Tables}

1 Empirical levels of asymptotic, bootstrap and MMC tests. . . . . . . . . . 20

2 Empirical power of asymptotic, bootstrap and MMC tests. . . . . . . . . . . 21

3 Empirical application on S\&P500 index. . . . . . . . . . . . . . 25 


\section{Introduction}

Modelling conditional heteroskedasticity is one of the central problems of financial econometrics. The two main families of models for that purpose consist of ARCH-type processes, originally introduced by Engle (1982), and stochastic volatility (SV) models proposed by Taylor (1994). Even though GARCH-type models are more widely used than SV models, the latter may be preferable for several reasons. First, SV models are directly connected to diffusion processes used in theoretical finance and allow for a volatility process that does not depend on observable variables. Second, as pointed out by Carnero, Peña and Ruiz (2004), kurtosis, volatility shock persistence and serial correlation of squared variables differ markedly between GARCH and autoregressive SV models (ARSV). This difference may explain why the estimated persistence is usually higher in GARCH than in Gaussian ARSV models, and why GARCH models often require leptokurtic conditional distributions.

GARCH models are relatively easy to estimate and remain much more popular than SV models. In particular, evaluating the likelihood function of GARCH models is simple compared to SV models, for which it is difficult to obtain a likelihood in closed form. This is a general feature of most nonlinear latent variable models, because the latent variables must be integrated out of the joint density for the observed and latent processes, leading to an integral of high dimensionality. As a result, maximum likelihood (ML) methods are prohibitively expensive from a computational viewpoint, and alternative methods appear to be required for applying such models. This fundamental difficulty may have prevented the widespread use of SV models and has made GARCH the model of choice in practice.

Nevertheless, much progress has been achieved on the estimation of SV models. Besides the quasi maximum likelihood approaches [Ruiz (1994)], or the generalized method of moments (GMM) procedures [Andersen and Sørensen (1996)], simulation-based estimation has become more attractive due to increasing computer power, and comprises: (1) indirect inference which has been used to estimate SV models by Monfardini (1998); (2) the efficient method of moments applied to SV models by Andersen, Chung and Sørensen (1999) and Chernov, Gallant, Ghysels and Tauchen (2003); (3) simulated maximum likelihood, which can be implemented in SV models using importance sampling; see Danielsson and Richard (1993), Danielsson (1994), Durham (2006, 2007). Bayesian techniques can also be applied in this context through computer-intensive methods, such as Markov Chain Monte Carlo (MCMC) methods, and appear to yield relatively good results; see Jacquier, Polson and Rossi (1994), Chib, Nardari and Shephard (2002).

All these studies focus on the estimation of SV models. Test problems have received much less attention. The available results on hypothesis testing for such models are rather incomplete and scattered. These include: GMM-based $t$-type tests on individual coefficients [Andersen and Sørensen (1996), Andersen et al. (1999)], and various specification tests such as tests for goodness of fit, diagnostic checking and model comparison; see Andersen and Sørensen (1996), Gallant, Hsieh and Tauchen (1997), Andersen et al. (1999), Durham (2006, 2007). A systematic discussion of hypothesis testing on SV model coefficients does not appear to be available. Further, even in parametric SV models, all the available test procedures are based on large-sample approximations and do not address non-regular problems which show up naturally in this context, such as testing 
the hypothesis of homoskedasticity against a SV model, or testing the hypothesis of a one-factor SV model against a two-factor SV model.

In this paper, we focus on hypothesis testing in parametric SV models. Our main objective is to develop both exact tests as well as asymptotically justified procedures that are markedly more reliable than those based on usual large-sample approximations, especially in the presence of nonregularities and non-standard asymptotic distributions. The proposed procedures are also designed to be computationally manageable.

Exploiting the fact that many SV models are parametric models involving only a finite number of unknown parameters, our basic outlook is to develop finite-sample simulation-based procedures as opposed to procedures based on establishing asymptotic distributions. For that purpose, we rely on extensions of the basic idea of Monte Carlo (MC) tests originally proposed by Dwass (1957) and Barnard (1963). When the distribution of a test statistic does not depend on (unknown) nuisance parameters, the technique of MC tests yields an exact test provided one can generate a few i.i.d. (or exchangeable) replications of the test statistic under the null hypothesis; for example, 19 replications are sufficient to get a test with level 0.05; see Dufour and Khalaf (2001). This technique can be extended to test statistics which depend on nuisance parameters by considering maximized Monte Carlo (MMC) tests; see Dufour (2006). MMC tests yield exact tests whenever the distribution of the test statistic can be simulated as a function of the nuisance parameters: no additional assumption on its distribution is needed. Further, computationally more tractable versions of this procedure, such as MMC tests on consistent set estimators of model nuisance parameters, provide asymptotically valid tests irrespective of the presence of non-regularities and non-standard asymptotic distributions, such as those associated with identification problems. Parametric bootstrap tests may also be interpreted as degenerate MMC tests, where the simulated $p$-value function is evaluated at a single nuisanceparameter point estimate. However, the asymptotic validity of the parametric bootstrap method requires stronger assumptions than the MMC procedure and it may fail to control the level of the test even asymptotically, especially in non-regular problems (where the MMC procedure remains valid). Thus, the only requirement for being able to use the MMC procedure is that one is able to generate artificial data from the test statistic or from the model without the need to study the asymptotic distribution of the test statistic nor even to establish its existence.

Even though the general approach proposed here can be applied to a wide array of setups and relatively general SV models, we focus here on a relatively simple log-normal SV model of order one with an autoregressive mean, which has been widely studied in the SV literature (usually in a more restricted form); see Jacquier et al. (1994), Danielsson (1994), Gallant et al. (1997). Further, for the sake of numerical tractability, we consider tests based on a simple two-step moment estimator which is available in closed form. This estimator is studied in detail in Dufour and Valéry (2006). However, the proposed test procedures by no way are restricted to being used with this specific estimator. In particular, any consistent estimator (GMM, SMM, indirect inference...) or even more efficient estimators such as the efficient method of moments or the simulated maximum likelihood estimator (SMLE) could be used instead (although the associated computational cost may be higher); see Danielsson (1994), Durham (2006, 2007). It is of interest to note here that the critical requirement for the validity of consistent set estimate maximized Monte Carlo tests (CSEMMC) is the consistency property for the estimate of the (nuisance) parameters, and not its efficiency. 
To be more specific, the contributions of the paper can be summarized as follows. First, we implement and compare the three standard test statistics, i.e. Wald-type, score-type and likelihoodratio-type tests based on the computationally simple moment estimator available in Dufour and Valéry (2006). Further, we propose a $C(\alpha)$-type test [see Neyman (1959), Dagenais and Dufour (1991)] which turns out to be relatively easy to implement in our framework and exhibits remarkably good size and power properties. Under standard regularity conditions, these test criteria follow asymptotic chi-square distributions under the null hypothesis. This holds, in particular, for linear hypotheses on the coefficients of the SV models and various (sufficiently smooth) nonlinear hypotheses. However, in view of the fact that the asymptotic distribution may be quite unreliable in finite samples, we suggest that such tests be implemented using MMC techniques (which are provably valid without further regularity conditions) and parametric bootstrapping. We also compare the performances of the different test criteria.

Second, we study in greater detail three relatively important special hypotheses in the context of the SV model, namely: (1) homoskedasticity (against the SV alternative); (2) the hypothesis of stochastic volatility without persistence (against persistence in stochastic volatility); (3) one-factor SV against a two-factor SV.

The first problem (testing homoskedasticity) is, of course, an important pre-test before trying to include a latent factor to drive the dynamics of the volatility process which makes its estimation much more complicated. However, moment conditions become locally singular in this case so that standard regularity conditions are not anymore applicable. Further, score-type test criteria [LM and $C(\alpha)$ ] and Wald-type are no longer computable in this case - at least without modification - so that they cannot be used. By contrast, bootstrap and MMC versions of LR-type tests appear to work well in this case.

The second problem [testing no persistence (or no clustering) in stochastic volatility] exhibits some similarities with testing homoskedasticity but remains different for two reasons: first, volatility is viewed as random but not persistent, which entails a non-Gaussian leptokurtic error distribution (while, in the homoskedastic case, the disturbances are i.i.d. Gaussian); second, the moment conditions do not imply singularities in the covariance matrices, which are invertible. Thus score-type test criteria [LM and $C(\alpha)$ ] and Wald-type can be computed in this case. In other words, testing no persistence (or no clustering) in stochastic volatility is a regular hypothesis for which standard asymptotic distributions are applicable. Through simulation evidence, however, we find that asymptotic critical values can lead to under-rejection rates in small samples, while the bootstrap procedure tends to over-reject. In contrast, the MMC method controls test level in all cases.

The third problem (testing one factor against two-factor SV) is motivated by the fact that standard SV models do not capture important features of asset returns distribution such as tail thickness; see Chernov et al. (2003) and Durham $(2006,2007)$. As a solution, a second factor in the volatility dynamics may account for tail behavior. Testing one-factor against two-factor SV introduces an unidentified parameter under the null hypothesis [as in Hansen (1996), Andrews (2001) and Dufour, Khalaf, Bernard and Genest (2004, section 3.2)], so that standard asymptotic regularity conditions do not hold. In addition to the identification problem, the Jacobian of the moment conditions fails to meet a required full-column rank condition. Therefore, under the third type of hypothesis, we have the most degenerate testing problem which combines both difficulties: an identification issue and 
a rank failure of the Jacobian. Thus, score-type criteria are not applicable because covariance matrices are singular and Wald-type tests become utterly unreliable [see Dufour (1997, 2003)]. Even bootstrapping appears to fail in this case. In contrast, we find that MMC-based LR-type tests work well for that problem. It is also interesting to note that developing and justifying solutions such as those based on the approach proposed by Davies (1977, 1987), Hansen (1996) and Andrews (2001) would require considerable additional theoretical work. In contrast, the MMC approach works transparently.

Fourth, we perform a Monte Carlo study to compare the finite-sample properties of the procedures considered. We make two important observations: (1) in regular test problems, $C(\alpha)$-type tests exhibit good performance, especially when they are implemented in a simulated approach (bootstrap or MMC); (2) in non-regular problems, the only procedure which is both widely applicable and allows one to control test level is the MMC-based LR-type test.

Fifth, the proposed procedures are applied to the Standard and Poor's Composite Price Index. For this series, we find evidence that stochastic volatility is present through a one-factor specification with strong persistence.

The paper is organized as follows. Section 2 sets the framework underlying the one-factor and two-factor SV models and reviews the estimation procedure used to implement the tests. The test criteria considered and the associated confidence sets are discussed in Section 3. In Section 4, we examine why some basic problems in this setup, such as testing homoskedasticity against SV or testing one-factor SV against two-factor SV, lead to non-regularities. In Section 5, we review the technique of Monte Carlo tests. Simulation results are presented in Section 6, while empirical results on the Standard and Poor's Composite Price Index 500 return series appear in Section 7. We conclude in Section 8.

\section{Framework}

\subsection{One-factor SV model}

The basic form of the stochastic volatility model we study here comes from Gallant et al. (1997). Let us denote by $y_{t}$ the variable of interest. For example, $y_{t}$ can denote the first difference over a short time interval, a day for instance, of the log-price of a financial asset traded on security markets.

Assumption 2.1 The process $\left\{y_{t}: t \in \mathbb{N}\right\}$ follows a stochastic volatility model of the type:

$$
\begin{gathered}
y_{t}-\mu_{y}=\sum_{i=1}^{L_{y}} c_{i}\left(y_{t-i}-\mu_{y}\right)+u_{t}, \\
u_{t}=\exp \left(w_{t} / 2\right) r_{y} z_{t}, \\
w_{t}=\sum_{j=1}^{L_{w}} a_{w j} w_{t-j}+r_{w} v_{t},
\end{gathered}
$$


where $\mu_{y},\left\{c_{j}\right\}_{j=1}^{L_{y}}, r_{y},\left\{a_{w j}\right\}_{j=1}^{L_{w}}$ and $r_{w}$ are unknown parameters and $s_{t}=\left(y_{t}, w_{t}\right)^{\prime}$ is initialized from its stationary distribution.

In the above model, (2.1) is the mean equation, while (2.3) is the volatility equation. We shall call the model represented by (2.1) - (2.3) the stochastic volatility model of order $L_{w}$ with autoregressive mean of order $L_{y}$ [ARSV $\left(L_{y}, L_{w}\right)$ for short]. The lag lengths of the autoregressive specifications used in the literature are typically short. Usual configurations include $\left(L_{y}, L_{w}\right)=(0,1)$, $(1,1)$ or $(2,2)$; see Andersen and Sørensen (1996), Gallant et al. (1997), and Andersen et al. (1999). An important special case of (2.1) - (2.3) consists in setting $c_{j}=a_{w j}=0, \forall j \geq 2$, and $\delta=\left(c, \theta^{\prime}\right)^{\prime}$ with $\theta=\theta_{1}$, where $\theta_{1}=\left(a_{w}, r_{y}, r_{w}\right)^{\prime}$. We then have:

$$
\begin{gathered}
y_{t}-\mu_{y}=c\left(y_{t-1}-\mu_{y}\right)+u_{t}, \quad|c|<1, \\
u_{t}=\left[r_{y} \exp \left(w_{t} / 2\right)\right] z_{t}, \\
w_{t}=a_{w} w_{t-1}+r_{w} v_{t}, \quad\left|a_{w}\right|<1 .
\end{gathered}
$$

Assumption 2.2 The vectors $\left(z_{t}, v_{t}\right)^{\prime}, t \in \mathbb{N}$ are i.i.d. according to a $N\left(0, I_{2}\right)$ distribution.

Assumption 2.3 The process $s_{t}=\left(y_{t}, w_{t}\right)^{\prime}$ is strictly stationary.

The $\operatorname{ARSV}\left(L_{y}, L_{w}\right)$ process is Markovian of order $L_{s}=\max \left(L_{y}, L_{w}\right)$. Let us denote by

$$
\delta=\left(\mu_{y}, c_{1}, \ldots, c_{L_{y}}, r_{y}, a_{w 1}, \ldots, a_{w L_{w}}, r_{w}\right)^{\prime}
$$

the parameter vector of the model. Here $\left\{y_{t}\right\}$ is observed, while $\left\{w_{t}\right\}$ is a latent variable. Accordingly, the joint density of the observation vector $y_{(T)}=\left(y_{1}, \ldots, y_{T}\right)$ is not available in closed form, for it requires evaluating an integral with dimension equal to the whole path of the latent volatilities. Let

$$
F\left(y_{1}, \ldots, y_{T}\right)=\mathrm{P}\left[Y_{1} \leq y_{1}, \ldots, Y_{T} \leq y_{T} \mid \delta\right] \equiv F_{0}\left(y_{(T)} \mid \delta\right)
$$

denote its unknown distribution function.

We shall now focus on the $\operatorname{ARSV}(1,1)$ model. To estimate it, we consider a two-step method whose first step consists in applying ordinary least squares (OLS) to the mean equation which yields a consistent estimate of the autoregressive parameter $c$ and of the mean parameter $\mu_{y}$, denoted by $\hat{c}, \hat{\mu}_{y}$ and the residuals $\hat{u}_{t} \equiv u_{t}(\hat{c})=y_{t}-\hat{\mu}_{y}-\hat{c}\left(y_{t-1}-\hat{\mu}_{y}\right)$. Then, we apply in a second step a method of moments to the residuals $\hat{u}_{t}$ to get the estimate of the parameter $\theta_{1}=\left(a_{w}, r_{y}, r_{w}\right)^{\prime}$ of the mean and volatility equations. ${ }^{1}$ Unlike the other estimators proposed in the financial literature for estimating SV models, this two-step moment estimator is easy to implement and available in closed form, an appealing feature for complicated latent variable models. Besides, its simplicity allows

\footnotetext{
${ }^{1}$ It is shown in Dufour and Valéry (2006) propositions 4.2 and 4.3 that replacing $c$ by $\hat{c}$ and using $\hat{u}_{t}=u_{t}(\hat{c})$ has no effect on the asymptotic variance of $\hat{\theta}$. An interesting and potentially useful feature of the asymptotic distribution of $\hat{\theta}$ stems from the fact that its covariance matrix does not depend on the distribution of the first-step conditional mean estimator and consequently no effect is passed on the test statistics. Otherwise, substituting estimators for parameters would affect the test results as shown in Pierce (1982).
} 
for simulation-based inference and will be further exploited to obtain simulated testing procedures. In the sequel we will focus on the particular case where $\mu_{y}=0$ but all the results still hold in the general case.

Under the assumptions $\mathbf{2 . 1}$ to $\mathbf{2 . 3}$, with $\mu_{y}=0$ and $c_{i}=a_{w i}=0, \forall i \geq 2$, the perturbation term $u_{t}$ has the following moments for positive even values of $j$ and $k$ :

$$
\begin{aligned}
& \mu_{k}\left(\theta_{1}\right) \equiv \mathrm{E}\left(u_{t}^{k}\right)=r_{y}^{k} \frac{k !}{2^{(k / 2)}(k / 2) !} \exp \left[\frac{k^{2}}{8} r_{w}^{2} /\left(1-a_{w}^{2}\right)\right], \\
& \mu_{j, k}\left(l \mid \theta_{1}\right) \equiv \mathrm{E}\left(u_{t}^{j} u_{t+l}^{k}\right) \\
&=r_{y}^{j+k} \frac{j !}{2^{(j / 2)}(j / 2) !} \frac{k !}{2^{(k / 2)}(k / 2) !} \exp \left[\frac{r_{w}^{2}}{8\left(1-a_{w}^{2}\right)}\left(j^{2}+k^{2}+2 j k a_{w}^{|l|}\right)\right] .
\end{aligned}
$$

Odd moments are equal to zero. In particular, for $j=2, j=4$ and $j=k=2$ and $l=1$, we have:

$$
\begin{gathered}
\mu_{2}\left(\theta_{1}\right)=\mathrm{E}\left(u_{t}^{2}\right)=r_{y}^{2} \exp \left[(1 / 2) r_{w}^{2} /\left(1-a_{w}^{2}\right)\right], \\
\mu_{4}\left(\theta_{1}\right)=\mathrm{E}\left(u_{t}^{4}\right)=3 r_{y}^{4} \exp \left[2 r_{w}^{2} /\left(1-a_{w}^{2}\right)\right], \\
\mu_{2,2}\left(1 \mid \theta_{1}\right)=\mathrm{E}\left[u_{t}^{2} u_{t-1}^{2}\right]=r_{y}^{4} \exp \left[r_{w}^{2} /\left(1-a_{w}\right)\right] ;
\end{gathered}
$$

see Dufour and Valéry (2006). Let

$$
\kappa=\frac{\mu_{4}\left(\theta_{1}\right)}{\mu_{2}^{2}\left(\theta_{1}\right)}
$$

be the kurtosis coefficient of the process. It is easy to see that $\kappa \geq 3$, with $\kappa>3$ as soon as $r_{w} \neq 0$ (i.e., when the volatility is not constant). Solving the above moment equations corresponding to $j=2, j=4$ and $l=1$ yields the following expressions: provided $\kappa>3$,

$$
a_{w}=\log \left[\frac{\mu_{2,2}\left(1 \mid \theta_{1}\right)}{\mu_{2}^{2}\left(\theta_{1}\right)}\right] / \log (\kappa / 3)
$$

hence

$$
r_{y}=\frac{3^{1 / 4} \mu_{2}\left(\theta_{1}\right)}{\mu_{4}\left(\theta_{1}\right)^{1 / 4}}=\left(\frac{3 \mu_{2}^{2}\left(\theta_{1}\right)}{\kappa}\right)^{1 / 4}, \quad r_{w}=\left[\left(1-a_{w}^{2}\right) \log (\kappa / 3)\right]^{1 / 2}, \quad \text { if } \kappa>3 .
$$

If $\kappa \leq 3$, the volatility is constant and it is natural to set

$$
a_{w}=r_{w}=0 \text { and } r_{y}=\sqrt{\mu_{2}\left(\theta_{1}\right)} \text { if } \kappa \leq 3 .
$$

Given the latter definitions, it is easy to compute a method-of-moment estimator for $\theta_{1}=$ $\left(a_{w}, r_{y}, r_{w}\right)^{\prime}$ on replacing the theoretical moments by sample counterparts based on the residuals $\hat{u}_{t}$. Let $\hat{\theta}$ denote the method-of-moments estimator of $\theta_{1}$. Typically, $\mathrm{E}\left(u_{t}^{2}\right), \mathrm{E}\left(u_{t}^{4}\right)$ and $\mathrm{E}\left(u_{t}^{2} u_{t-1}^{2}\right)$ 
are approximated by:

$$
\hat{\mu}_{2}=\frac{1}{T} \sum_{t=1}^{T} \hat{u}_{t}^{2} \quad \hat{\mu}_{4}=\frac{1}{T} \sum_{t=1}^{T} \hat{u}_{t}^{4}, \quad \hat{\mu}_{2,2}(1)=\frac{1}{T-1} \sum_{t=2}^{T} \hat{u}_{t}^{2} \hat{u}_{t-1}^{2}
$$

respectively. This yields the following estimators of the stochastic volatility coefficients:

$$
\begin{aligned}
& \hat{a}_{w}=\left\{\begin{aligned}
\Delta & \text { if } \tilde{a}_{w}>\Delta, \\
\tilde{a}_{w} & \text { if }\left|\tilde{a}_{w}\right| \leq \Delta, \\
-\Delta & \text { if } \tilde{a}_{w}<-\Delta
\end{aligned}\right. \\
& \hat{r}_{y}=\left(3 \hat{\mu}_{2}^{2} / \hat{\kappa}\right)^{1 / 4} \quad \text { if } \hat{\kappa}>3, \\
& =\hat{\mu}_{2}^{1 / 2} \quad \text { if } \hat{\kappa} \leq 3, \\
& \begin{array}{rlr}
\hat{r}_{w} & =\left[\left(1-\hat{a}_{w}^{2}\right) \log (\hat{\kappa} / 3)\right]^{1 / 2} & \text { if } \hat{\kappa}>3, \\
& =0 & \text { if } \hat{\kappa} \leq 3,
\end{array}
\end{aligned}
$$

where $\hat{\kappa}=\hat{\mu}_{4} / \hat{\mu}_{2}^{2}$ and

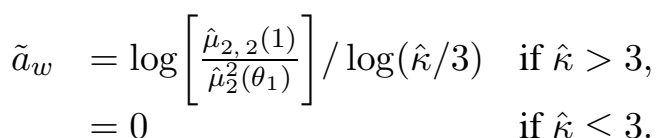

In (2.18), $\Delta$ is a number close to one which is used to bound the estimator away from the stationary boundary. This is important to avoid numerical instability. In the simulations and application below, we used $\Delta=0.99$, but a value closer to one could be considered. Under the assumptions of the model, the restriction $\hat{\kappa} \geq 3$ must hold with probability converging to one. Provided $\left|a_{w}\right|<\Delta$, the estimator $\hat{\theta}=\left[\hat{a}_{w}, \hat{r}_{y}, \hat{r}_{w}\right]^{\prime}$ is consistent and asymptotically normally distributed; see Dufour and Valéry (2006) for a detailed presentation of its asymptotic properties. ${ }^{2}$ However, the Monte Carlo tests procedure used later in the paper is restricted by no way to this specific estimator. Thus, any consistent estimator (e.g., GMM, SMM) or even more efficient estimators such as the efficient method of moments (see Chernov et al. (2003)) or the simulated maximum likelihood estimator (SMLE) could be used instead (although the associated computational cost may be higher), see Durham $(2006,2007)$ and may induce power gains.

\subsection{Two-factor SV model}

A simple single-factor SV model appears to be sufficient to capture the salient properties of volatility such as randomness and persistence. It is the shape of the conditional distribution of financial returns which constitutes the problem; see Chernov et al. (2003) and Durham (2006, 2007). Standard SV models cannot match the high conditional kurtosis of returns (tail thickness) documented in the

\footnotetext{
${ }^{2}$ The estimated covariance matrix estimated is is $\left(\hat{J}^{\prime} \hat{I}^{-1} \hat{J}\right)^{-1}$ with $\hat{J}=J(\hat{\theta}), J(\theta)=\frac{\partial \mu}{\partial \theta^{\prime}}$ and $\hat{I}=\hat{\Omega}_{*}$, where $\hat{\Omega}_{*}$ is defined by (3.3) and (3.4). Standard errors are the square roots of the corresponding variances from this covariance matrix.
} 
financial literature, for example in the case of equities. Trying to capture nonlinearities in financial returns has important implications for risk management and option pricing.

Consequently, we also consider a two-factor specification driving the dynamics of the volatility process of the following form:

$$
\begin{aligned}
y_{t}-\mu_{y} & =c\left(y_{t-1}-\mu_{y}\right)+u_{t}, \quad|c|<1, \\
u_{t} & =\left[r_{y} \exp \left(w_{t} / 2+\eta_{t} / 2\right)\right] z_{t} \\
w_{t} & =a_{w} w_{t-1}+r_{w} v_{1 t}, \quad\left|a_{w}\right|<1 \\
\eta_{t} & =a_{\eta} \eta_{t-1}+r_{\eta} v_{2 t}, \quad\left|a_{\eta}\right|<1
\end{aligned}
$$

$\left(z_{t}, v_{1 t}, v_{2 t}\right)$ are i.i.d. Gaussian vectors such that $z_{t} \sim N(0,1)$ and

$$
\left(v_{1 t}, v_{2 t}\right) \sim N\left(0, \Sigma_{v}\right), \quad \Sigma_{v}=\left[\begin{array}{cc}
1 & \rho_{12} \\
\rho_{12} & 1
\end{array}\right], \quad \mathrm{E}\left[\left(v_{1 t}, v_{2 t}\right) z_{t}\right]=0 .
$$

We shall call the above model represented by equations (2.22) - (2.26) the autoregressive stochastic volatility model with two factors. Let $\theta_{2}=\left(a_{w}, r_{y}, r_{w}, a_{\eta}, r_{\eta}, \rho_{12}\right)^{\prime}$ denote the parameter corresponding to the two-factor SV model. We derive the moment conditions used in a just-identified GMM framework, which are stated in the proposition below.

Proposition 2.4 MOMENTS OF THE TWO-FACTOR SV PROCESS. Under the assumptions (2.22) to (2.26), we have for positive even values of $j$ and $k$ :

$$
\begin{gathered}
\mathrm{E}\left(u_{t}^{k}\right)=r_{y}^{k} \frac{k !}{2^{(k / 2)}(k / 2) !} \exp \left[\frac{k^{2}}{8} r_{w}^{2} /\left(1-a_{w}^{2}\right)+\frac{k^{2}}{8} r_{\eta}^{2} /\left(1-a_{\eta}^{2}\right)+\frac{k^{2}}{4} \frac{r_{w} r_{\eta} \rho_{12}}{1-a_{w} a_{\eta}}\right], \\
\begin{aligned}
\mathrm{E}\left[u_{t}^{j} u_{t+l}^{k}\right]= & r_{y}^{j+k} \frac{j !}{2^{(j / 2)}(j / 2) !} \frac{k !}{2^{(k / 2)}(k / 2) !} \exp \left[\frac{r_{w}^{2}}{8\left(1-a_{w}^{2}\right)}\left(j^{2}+k^{2}+2 j k a_{w}^{|l|}\right)\right. \\
& +\frac{r_{\eta}^{2}}{8\left(1-a_{\eta}^{2}\right)}\left(j^{2}+k^{2}+2 j k a_{\eta}^{|l|}\right) \\
& \left.+\frac{1}{8}\left(2 j^{2}+2 k^{2}+2 j k a_{\eta}^{|l|}+2 j k a_{w}^{|l|}\right) \frac{r_{w} r_{\eta} \rho_{12}}{1-a_{w} a_{\eta}}\right] .
\end{aligned}
\end{gathered}
$$

The proof of this proposition is given in Appendix A. In particular, for $j=2,4,6$ and $j=k$, the above formulae yield the following moments:

$$
\begin{aligned}
\mathrm{E}\left(u_{t}^{2}\right) & =r_{y}^{2} \exp \left(\frac{1}{2} \frac{r_{w}^{2}}{1-a_{w}^{2}}+\frac{1}{2} \frac{r_{\eta}^{2}}{1-a_{\eta}^{2}}+\frac{r_{w} r_{\eta} \rho_{12}}{1-a_{w} a_{\eta}}\right) \equiv \mu_{2}\left(\theta_{2}\right), \\
\mathrm{E}\left(u_{t}^{4}\right) & =3 r_{y}^{4} \exp \left(\frac{2 r_{w}^{2}}{1-a_{w}^{2}}+\frac{2 r_{\eta}^{2}}{1-a_{\eta}^{2}}+\frac{4 r_{w} r_{\eta} \rho_{12}}{1-a_{w} a_{\eta}}\right) \equiv \mu_{4}\left(\theta_{2}\right),
\end{aligned}
$$




$$
\begin{gathered}
\mathrm{E}\left(u_{t}^{6}\right)=15 r_{y}^{6} \exp \left(\frac{9}{2} \frac{r_{w}^{2}}{1-a_{w}^{2}}+\frac{9}{2} \frac{r_{\eta}^{2}}{1-a_{\eta}^{2}}+\frac{9 r_{w} r_{\eta} \rho_{12}}{1-a_{w} a_{\eta}}\right) \equiv \mu_{6}\left(\theta_{2}\right), \\
\mathrm{E}\left[u_{t}^{2} u_{t-1}^{2}\right]=r_{y}^{4} \exp \left(\frac{\sigma^{2}}{2}\right)=\mu_{2,2}\left(1 \mid \theta_{2}\right), \\
\mathrm{E}\left[u_{t}^{4} u_{t-1}^{4}\right]=9 r_{y}^{8} \exp \left(2 \sigma^{2}\right)=\mu_{4,4}\left(1 \mid \theta_{2}\right), \\
\mathrm{E}\left[u_{t}^{6} u_{t-1}^{6}\right]=225 r_{y}^{12} \exp \left(\frac{9}{2} \sigma^{2}\right)=\mu_{6,6}\left(1 \mid \theta_{2}\right),
\end{gathered}
$$

where

$$
\begin{aligned}
\sigma^{2} \equiv \operatorname{Var}\left(w_{t}+\eta_{t}+w_{t-1}+\eta_{t-1}\right)= & \frac{2 r_{w}^{2}}{1-a_{w}^{2}}+\frac{2 r_{\eta}^{2}}{1-a_{\eta}^{2}}+\frac{4 r_{w} r_{\eta} \rho_{12}}{1-a_{w} a_{\eta}}+\frac{2 a_{w} r_{w}^{2}}{1-a_{w}^{2}} \\
& +\frac{2 a_{w} r_{w} r_{\eta} \rho_{12}}{1-a_{w} a_{\eta}}+\frac{2 a_{\eta} r_{w} r_{\eta} \rho_{12}}{1-a_{w} a_{\eta}}+\frac{2 a_{\eta} r_{\eta}^{2}}{1-a_{\eta}^{2}}
\end{aligned}
$$

These moment conditions constitute a just-identified GMM setup we shall use below in order to test the number of SV factors in the volatility process. The associated estimators, however, are not available in closed form, in contrast with the one-factor setup. But the moment conditions (2.28) - (2.33) yield a GMM estimator in the usual way through nonlinear optimization techniques.

It is important to note that another set of moment conditions (larger or simply different) could be used to estimate the two-factor model. This might lead to more precise estimates and eventually

more powerful tests. Finding better or "optimal" moment conditions goes beyond the scope the present paper. But the general testing approach proposed below remains applicable if different sets of moment conditions are employed.

\section{Test statistics and confidence sets}

We are concerned with testing a null hypothesis of the form:

$$
H_{0}\left(\psi_{0}\right): \psi(\theta)=\psi_{0} .
$$

Further, we assume that the derivative of the constraints

$$
P(\theta)=\frac{\partial \psi}{\partial \theta^{\prime}}
$$

has full row rank, let $\hat{\theta}$ be the unrestricted estimator and $\hat{\theta}_{0}$ the constrained estimator obtained by minimizing the following criterion

$$
M_{T}^{*}(\theta) \equiv\left[\bar{g}_{T}\left(\hat{U}_{T}\right)-\mu(\theta)\right]^{\prime} \hat{\Omega}_{*}^{-1}\left[\bar{g}_{T}\left(\hat{U}_{T}\right)-\mu(\theta)\right]
$$


where $\bar{g}_{T}\left(\hat{U}_{T}\right)$ denotes the vector of empirical moments based on the residual vector $\hat{U}_{T}$ corresponding to $\mu(\theta)$. $\hat{\Omega}_{*}$ denotes a consistent estimator of $\Omega_{*}$,

$$
\Omega_{*}=\lim _{T \rightarrow \infty} \mathrm{E}\left\{T\left[\bar{g}_{T}\left(U_{T}\right)-\mu\left(\theta_{0}\right)\right]\left[\bar{g}_{T}\left(U_{T}\right)-\mu\left(\theta_{0}\right)\right]^{\prime}\right\},
$$

with $\theta_{0}$ denoting the true value of $\theta$. A consistent estimator $\hat{\Omega}_{*}$ which accounts for the autocorrelation between $u_{t}^{2}, u_{t}^{4}, \ldots$, can easily be obtained using a Bartlett kernel:

$$
\hat{\Omega}_{*}=\hat{\Gamma}_{0}+\sum_{k=1}^{K(T)}\left(1-\frac{k}{K(T)+1}\right)\left(\hat{\Gamma}_{k}+\hat{\Gamma}_{k}^{\prime}\right)
$$

where

$$
\hat{\Gamma}_{k}=\frac{1}{T} \sum_{t=k+1}^{T}\left[g_{t-k}\left(\hat{U}_{T}\right)-\mu(\tilde{\theta})\right]\left[g_{t}\left(\hat{U}_{T}\right)-\mu(\tilde{\theta})\right]^{\prime}
$$

$\tilde{\theta}$ is a consistent estimator of $\theta, g_{t}\left(\hat{U}_{T}\right)=\left[\hat{u}_{t}^{2}, \hat{u}_{t}^{4}, \hat{u}_{t}^{2} \hat{u}_{t-1}^{2}\right]^{\prime}$ for the SV model (2.4) - (2.6), and $g_{t}\left(\hat{U}_{T}\right)=\left[\hat{u}_{t}^{2}, \hat{u}_{t}^{4}, \hat{u}_{t}^{6}, \hat{u}_{t}^{2} \hat{u}_{t-1}^{2}, \hat{u}_{t}^{4} \hat{u}_{t-1}^{4}, \hat{u}_{t}^{6} \hat{u}_{t-1}^{6}\right]^{\prime}$ for the SV model (2.22)-(2.26). In a justidentified framework, the choice of weight matrix $\hat{\Omega}_{*}^{-1}$ is irrelevant.

The Wald-type statistic is defined as

$$
\xi_{T}^{W}=T\left[\psi(\hat{\theta})-\psi_{0}\right]^{\prime}\left[\hat{P}\left(\hat{J}^{\prime} \hat{I}^{-1} \hat{J}\right)^{-1} \hat{P}^{\prime}\right]^{-1}\left[\psi(\hat{\theta})-\psi_{0}\right]
$$

where $\hat{P}=P(\hat{\theta}), \hat{I}=I(\hat{\theta})=\Omega_{*}(\hat{\theta}), \hat{J}=J(\hat{\theta})=\frac{\partial \mu}{\partial \theta^{\prime}}(\hat{\theta})$. The score-type statistic is based on the gradient of the objective function with respect to $\theta$ evaluated at the constrained estimator. This gradient is

$$
\mathcal{D}_{T}=\frac{\partial \mu^{\prime}}{\partial \theta}\left(\hat{\theta}_{0}\right) \hat{\Omega}_{*}^{-1}\left[\mu\left(\hat{\theta}_{0}\right)-\bar{g}_{T}\left(\hat{U}_{T}\right)\right]=\hat{J}_{0}^{\prime} \hat{I}_{0}^{-1}\left[\mu\left(\hat{\theta}_{0}\right)-\bar{g}_{T}\left(\hat{U}_{T}\right)\right]
$$

where $\hat{I}_{0}=I\left(\hat{\theta}_{0}\right)=\Omega_{*}\left(\hat{\theta}_{0}\right), \hat{J}_{0}=J\left(\hat{\theta}_{0}\right)=\frac{\partial \mu}{\partial \theta^{\prime}}\left(\hat{\theta}_{0}\right)$, and the test statistic is

$$
\xi_{T}^{S}=T \mathcal{D}_{T}^{\prime}\left(\hat{J}_{0}^{\prime} \hat{I}_{0}^{-1} \hat{J}_{0}\right)^{-1} \mathcal{D}_{T}=T\left[\mu\left(\hat{\theta}_{0}\right)-\bar{g}_{T}\left(\hat{U}_{T}\right)\right]^{\prime} \hat{W}_{0}\left[\mu\left(\hat{\theta}_{0}\right)-\bar{g}_{T}\left(\hat{U}_{T}\right)\right],
$$

with $\hat{W}_{0}=\hat{I}_{0}^{-1} \hat{J}_{0}\left(\hat{J}_{0}^{\prime} \hat{I}_{0}^{-1} \hat{J}_{0}\right)^{-1} \hat{J}_{0}^{\prime} \hat{I}_{0}^{-1}$. Finally, the difference between the restricted and unrestricted optimal values of the objective function is called the LR-type statistic:

$$
\xi_{T}^{C}=T\left[M_{T}^{*}\left(\hat{\theta}_{0}\right)-M_{T}^{*}(\hat{\theta})\right] .
$$

Provided

$$
T\left[M_{T}^{*}(\theta)-M_{T}(\theta)\right] \underset{T \rightarrow \infty}{\longrightarrow} 0
$$

uniformly in a neighborhood of the true distribution, where

$$
M_{T}(\theta) \equiv\left[\bar{g}_{T}\left(\hat{U}_{T}\right)-\mu(\theta)\right]^{\prime} \Omega_{*}^{-1}\left[\bar{g}_{T}\left(\hat{U}_{T}\right)-\mu(\theta)\right],
$$


the three test statistics $\xi_{T}^{W}, \xi_{T}^{S}$ and $\xi_{T}^{C}$ follow a $\chi^{2}(\nu)$ distribution asymptotically under the null hypothesis (with standard regularity conditions), where $\nu$ is the number of constraints.

We also consider the $C(\alpha)$-type test statistic defined by

$$
P C\left(\tilde{\theta}_{0}\right)=T\left[\mu\left(\tilde{\theta}_{0}\right)-\bar{g}_{T}\left(\hat{U}_{T}\right)\right]^{\prime} \tilde{W}_{0}\left[\mu\left(\tilde{\theta}_{0}\right)-\bar{g}_{T}\left(\hat{U}_{T}\right)\right]
$$

where

$$
\tilde{W}_{0}=\tilde{I}_{0}^{-1} \tilde{J}_{0}\left(\tilde{J}_{0}^{\prime} \tilde{I}_{0}^{-1} \tilde{J}_{0}\right)^{-1} \tilde{P}_{0}^{\prime}\left[\tilde{P}_{0}\left(\tilde{J}_{0}^{\prime} \tilde{I}_{0}^{-1} \tilde{J}_{0}\right)^{-1} \tilde{P}_{0}^{\prime}\right]^{-1} \tilde{P}_{0}\left(\tilde{J}_{0}^{\prime} \tilde{I}_{0}^{-1} \tilde{J}_{0}\right)^{-1} \tilde{J}_{0}^{\prime} \tilde{I}_{0}^{-1}
$$

with $\tilde{J}_{0}=J\left(\tilde{\theta}_{0}\right)=\frac{\partial \mu}{\partial \theta^{\prime}}\left(\tilde{\theta}_{0}\right), \tilde{I}_{0}=I\left(\tilde{\theta}_{0}\right)=\Omega^{*}\left(\tilde{\theta}_{0}\right)$, and $\tilde{P}_{0}=P\left(\tilde{\theta}_{0}\right) . \tilde{\theta}_{0}$ is any root-n consistent estimator of $\theta$ that satisfies $\psi\left(\tilde{\theta}_{0}\right)=0$. Below, for the $\operatorname{ARSV}(1,1)$ model, $\tilde{\theta}_{0}$ will be obtained by imposing the constraints in the analytic expressions of the unrestricted method-of-moments estimator $\hat{\theta}$ defined by (2.18) - (2.21), yielding a consistent restricted estimator without the need to perform a nonlinear optimization. Again, under standard regularity conditions, the $C(\alpha)$-type test statistic is asymptotically distributed like a $\chi^{2}(\nu)$ variable under the null hypothesis; see Davidson and MacKinnon (1993, page 619) and Dufour and Trognon (2001, Proposition 3.1).

In the simulations, we will focus on parametric functions of the form

$$
\psi(\theta)=(1,0)\left(\begin{array}{c}
\theta_{s 1} \\
\theta_{s 2}
\end{array}\right)=\theta_{s 1}
$$

in which case the null hypothesis $H_{0}\left(\psi_{0}\right): \psi(\theta)=\psi_{0}$ simplifies to $H_{0}\left(\psi_{0}\right): \theta_{s 1}=\theta_{s 1}^{0}$. For example, we may have $\theta_{s 1} \equiv a_{w}, \theta_{s 1} \equiv\left(a_{w}, r_{w}\right)^{\prime}$.

Tests may also be used to build confidence sets for model parameters. Let $S_{0}=S\left(\psi_{0}, y_{(T)}\right)$ denote one of the four previous test statistics computed from the sample points $y_{(T)}=\left(y_{1}, \ldots, y_{T}\right)$ and under the hypothesis $H_{0}\left(\psi_{0}\right): \psi(\theta)=\psi_{0}$. If the acceptance region of the test for $H_{0}\left(\psi_{0}\right)$ : $\psi(\theta)=\psi_{0}$ has the form

$$
A\left(\psi_{0}\right)=\left\{y_{(T)}=\left(y_{1}, \ldots, y_{T}\right) \in \mathcal{Y}: S\left(\psi_{0}, y_{(T)}\right) \leq c(\alpha)\right\}
$$

where $c(\alpha)$ is the critical point for a test with level $\alpha$, the corresponding confidence set is the set of values $\psi_{0}$ which are not rejected by such tests:

$$
C_{\psi}\left(y_{(T)}\right)=\left\{\psi_{0}: S\left(\psi_{0}, y_{(T)}\right) \leq c(\alpha)\right\}=\left\{\psi_{0}: G\left[S\left(\psi_{0}, y_{(T)}\right)\right] \geq \alpha\right\}
$$

where $G(\cdot)$ denotes the $p$-value function. These sets are connected to each other by the equivalence

$$
y_{(T)} \in A\left(\psi_{0}\right) \Leftrightarrow \psi_{0} \in C\left(y_{(T)}\right)
$$

$>$ From the level condition

$$
\mathrm{P}_{F}\left[Y \notin A\left(\psi_{0}\right)\right] \leq \alpha, \quad \forall F \in \mathcal{H}_{0}\left(\psi_{0}\right)
$$


it follows that

$$
\begin{gathered}
\mathrm{P}_{F}\left[Y \in A\left(\psi_{0}\right)\right] \geq 1-\alpha, \quad \forall F \in \mathcal{H}_{0}\left(\psi_{0}\right), \\
\mathrm{P}_{F}\left[\psi_{0} \in C(Y)\right]=\mathrm{P}_{F}\left[Y \in A\left(\psi_{0}\right)\right] \geq 1-\alpha, \quad \forall F \in \mathcal{H}_{0}\left(\psi_{0}\right), \quad \forall \psi_{0} \in \Psi_{0},
\end{gathered}
$$

and

$$
\mathrm{P}_{F}[\psi(\theta) \in C(Y)] \geq 1-\alpha, \quad \text { for all } \theta,
$$

which means that $C_{\psi}(Y)$ is a confidence set with level $1-\alpha$ for $\psi(\theta)$.

Following this methodology, confidence sets can be built for any parameter of the volatility process by finding the values of the parameter for which the $p$-value function is greater than or equal to $\alpha$, yielding a confidence set with level $1-\alpha$.

\section{Non-regular problems}

We investigate in this section three interesting test problems. The first one consists in testing the homoskedasticity hypothesis $\left(a_{w}=r_{w}=0\right)$ against the SV alternative; the second one involves testing a SV hypothesis without persistence $\left(a_{w}=0\right)$ against persistence in SV; the third problem is a test of one-factor $\mathrm{SV}\left(a_{\eta}=r_{\eta}=0\right)$ against two-factor SV. Although these hypotheses are quite relevant in the context of SV models, they raise statistical difficulties. Indeed, under such null hypotheses, standard regularity conditions turn out to be violated, thus making the problems nonregular (although in somewhat different ways), so that the standard distributional theory presented in Section 3 does not apply anymore.

Let us consider first the problem of testing homoskedasticity $\left(a_{w}=r_{w}=0\right)$, which can be written $\psi(\theta)=0$ with $\psi(\theta)=\left(a_{w}, r_{w}\right)^{\prime}$. In this case, we have two restrictions, and the matrix

$$
P(\theta)=\frac{\partial \psi}{\partial \theta^{\prime}}=\left(\begin{array}{lll}
1 & 0 & 0 \\
0 & 1 & 0
\end{array}\right)
$$

has full rank two, so it appears to be regular. However, the Jacobian of the moment conditions (i.e., the derivative matrix of the moments with respect to the SV coefficients) does not have full rank when evaluated at a point that satisfies the null hypothesis: on using the analytical expressions for the derivatives of $\mu(\theta)$ with respect to $\theta=\left(a_{w}, r_{w}, r_{y}\right)$, as given in Appendix $\mathrm{B}$, we see that

$$
\frac{\partial \mu}{\partial \theta^{\prime}}=\left[\begin{array}{rrr}
0 & 0 & 2 r_{y} \\
0 & 0 & 12 r_{y}^{3} \\
0 & 0 & 4 r_{y}^{3}
\end{array}\right]
$$

when $a_{w}=r_{w}=0$, so that the Jacobian $\partial \mu / \partial \theta^{\prime}$ has at most rank one (instead of three in the fullrank case). But GMM identification requires a full-rank Jacobian; see Newey and McFadden (1994, p. 2127). An important regularity condition is violated. This raises estimation difficulties and was handled by redefining the estimator in this case: we set $a_{w}=r_{w}=0$ and $r_{y}=\sqrt{\mu_{2}\left(\theta_{1}\right)}$ when $\kappa \leq$ 3 ; see equations (2.16) - (2.21) above. 
A violation of the rank condition entails that the score-based statistics [the score and $C(\alpha)$-type statistics] involve non-invertible matrices, so that these tests are not applicable (at least, without modifications). Further, $\partial \mu / \partial \theta^{\prime}$ typically has full rank when it is evaluated at a point that does not satisfy the null hypothesis, for example at an unrestricted point estimate of $\theta$, as in Waldtype statistics. Therefore, the rank of $\partial \mu / \partial \theta^{\prime}$, when evaluated at an unrestricted point estimate of $\theta$, generally exceeds the rank of $\partial \mu / \partial \theta^{\prime}$ evaluated at the true $\theta$ when $a_{w}=r_{w}=0$ holds. This is again a violation of a standard regularity condition, and the Wald statistic has a non-regular asymptotic distribution; see Andrews (1987) and Lütkepohl and Burda (1997).

Second, the problem of testing no persistence in the SV displays some similarities with testing homoskedasticity $\left(a_{w}=r_{w}=0\right)$. Indeed, under the null hypothesis $a_{w}=0$,

$$
\operatorname{Var}\left(u_{t}\right)=r_{y}^{2} \mathrm{E}\left[\exp \left(r_{w} v_{t}\right)\right] \mathrm{E}\left(z_{t}^{2}\right)=r_{y}^{2} \exp \left(r_{w}^{2} / 2\right)
$$

is invariant over time. However, both hypotheses $\left(a_{w}=0\right.$ and $\left.a_{w}=r_{w}=0\right)$ have important statistical implications for estimation as well as for the rank of the Jacobian matrix associated with $\mu(\theta)$. Two points are worth being emphasized here.

1. Under $a_{w}=0$, the volatility is stochastic, whereas it is deterministic when $a_{w}=r_{w}=0$. When $a_{w}=0$, the kurtosis coefficient $\kappa$ always remains greater than 3 for $r_{w}>0[\kappa=$ $\left.3 \exp \left(r_{w}^{2}\right)\right]$ : the scale factor $\exp \left(r_{w} v_{t}\right)$ is lognormal, so $u_{t}$ has a leptokurtic distribution. In view of this distinction, the estimator $\hat{\theta}$ is defined differently under the two hypotheses: under $a_{w}=0$, it is defined by substituting sample analogs in equations (2.14) - (2.15), whereas under $a_{w}=r_{w}=0$ (so that $\kappa=3$ ), we set $a_{w}=r_{w}=0$ and use the sample analog of $r_{y}=\sqrt{\mu_{2}\left(\theta_{1}\right)}$.

2. Under $a_{w}=0$, the Jacobian

$$
\frac{\partial \mu}{\partial \theta^{\prime}}=\left[\begin{array}{lll}
0 & r_{w} r_{y}^{2} \exp \left[1 / 2\left(r_{w}^{2}\right)\right] & 2 r_{y} \exp \left[1 / 2\left(r_{w}^{2}\right)\right] \\
0 & 12 r_{w} r_{y}^{4} \exp \left(2 r_{w}^{2}\right) & 12 r_{y}^{3} \exp \left(2 r_{w}^{2}\right) \\
r_{w}^{2} r_{y}^{4} \exp \left(r_{w}^{2}\right) & 2 r_{w} r_{y}^{4} \exp \left(r_{w}^{2}\right) & 4 r_{y}^{3} \exp \left(r_{w}^{2}\right)
\end{array}\right]
$$

has full-column rank almost everywhere, except precisely when $r_{w}=0$ as well (provided $r_{y} \neq 0$ ). Indeed, $r_{w}$ and $r_{y}$ are identifiable when $a_{w}=0$, because they are uniquely determined by the second and fourth moments of $u_{t}$ [see equation (2.15)]. However, values of $a_{w}$ close to zero may lead to irregular statistical properties (similar to what occurs under the homoskedasticity hypothesis).

Third, when testing one-factor SV $\left(a_{\eta}=r_{\eta}=0\right)$ against a two-factor SV, the correlation parameter $\rho_{12}=\operatorname{corr}\left(v_{1 t}, v_{2 t}\right)$ becomes unidentified under the null hypothesis. Then, as in (4.1), the Jacobian of the moment conditions (2.28) - (2.33) does not have full-column rank when $a_{\eta}=r_{\eta}=0$; see Appendix C. This again creates a singularity, and standard regularity conditions are violated. In particular, score-type statistics are not applicable (without modification), and the distributions of all test criteria may be affected. This problem is similar to the one originally studied by Davies (1977, 1987). Similar situations arise when one tries to test significance for both AR and MA para- 
meter simultaneously in an ARMA model and also for both coefficients in standard $\operatorname{GARCH}(1,1)$ model. $^{3}$

More generally, it is well known that identification failure - or conditions close to identification failure (such as weak instruments) - can make methods based on Wald-type statistics fundamentally invalid - even though they remain computable - and require important adjustments to critical values used with other test statistics, such as LR-type statistics; see Dufour $(1997,2003)$ and Stock, Wright and Yogo (2002). In Section 6, we present simulation evidence which shows this is indeed the case here for LR-type statistics. Although adjustments - such as bounds similar to those considered by Davies (1977, 1987), Dufour (1989), Hansen (1996), Andrews (2001), Dufour and Khalaf (2002) - might be developed, justifying and applying such methods here would require a considerable theoretical effort.

In this paper, we take a different approach based on using a general method which is completely immune to possible singularities and identification problems, as well as relatively easy to apply. If no nuisance parameter were present in the distribution of the test statistic under the null hypothesis, an exact test could indeed easily be obtained by applying the technique of MC tests [as done, for example, in Dufour et al. (2004) for the Bera and Ra (1995) test against ARCH-M heteroskedasticity]. However, the nuisance-parameter problem does not go away here, and we propose to solve it by using the technique of maximized Monte Carlo tests; see Dufour (2006). We will now describe succinctly this method.

\section{Monte Carlo tests}

The technique of Monte Carlo tests was originally been proposed by Dwass (1957) for implementing permutation tests and did not involve nuisance parameters. This technique was also independently proposed by Barnard (1963); for a review, see Dufour and Khalaf (2001). It has the great attraction of providing exact (randomized) tests based on any statistic whose finite-sample distribution may be intractable but can be simulated. We briefly review the methodology of Monte Carlo tests covering both cases, first without nuisance parameters and then with nuisance parameters. The technique of Monte Carlo tests provides a simple method allowing one to replace the unknown or intractable theoretical distribution $F(y \mid \delta)$, where $\delta=\left(c, \theta^{\prime}\right)^{\prime}$, by its sample analogue based on the statistics $S_{1}(\delta), \ldots, S_{N}(\delta)$ simulated under the null hypothesis.

For the sake of clarity, let us first consider the case where no nuisance parameter is present.

1. Using the observed sample, we calculate the relevant statistic $S_{0}$.

2. Using draws under $H_{0}$, we generate $\mathrm{N}$ simulated samples $S_{1}, \ldots, S_{N}$.

\footnotetext{
${ }^{3}$ For various econometric examples and discussions of this problem, the reader may consult Andrews and Ploberger (1995), Bera and Ra (1995), Hansen (1996), Andrews (2001), Bera, Ra and Sarkar (1998), Andrews (2001) and Dufour et al. (2004).
} 
3. Then we consider the following simulated survival function

$$
\hat{G}_{N}[y ; S(N)]=\frac{1}{N} \sum_{i=1}^{N} s\left(S_{i}-y\right)
$$

and the associated $p$-value function

$$
\hat{p}_{N}(y)=\frac{N \hat{G}_{N}(y)+1}{N+1}
$$

where $s(x)=1$ if $x \geq 0$, and $s(x)=0$ if $x<0$. If the distribution of $S$ is continuous and $N$ is chosen so that $\alpha(N+1)$ is an integer, then

$$
\mathrm{P}\left[\hat{p}_{N}\left(S_{0}\right) \leq \alpha\right]=\alpha, \text { under } H_{0},
$$

which means that the critical region $\hat{p}_{N}\left(S_{0}\right) \leq \alpha$ has size $\alpha$.

In most econometric models, the relevant case is the one where the distribution of the test statistic depends on nuisance parameters. To deal with this complication, the MC test procedure can be modified as follows, where $\bar{\delta}$ represents the true parameter vector.

1. To test the null hypothesis

$$
H_{0}: \bar{\delta} \in \Omega_{0},
$$

we use first the observed sample to calculate the relevant statistic denoted by $S_{0}$.

2. For each $\delta \in \Omega_{0}$, we generate $N$ replications of $S: S_{1}(\delta), \ldots, S_{N}(\delta)$.

3. Using these simulations we compute the corresponding simulated $p$-value function:

$$
\hat{p}_{N}[y \mid \delta]=\frac{N \hat{G}_{N}[y \mid \delta]+1}{N+1} .
$$

4. The $p$-value function $\hat{p}_{N}\left[S_{0} \mid \delta\right]$ as a function of $\delta$ is maximized over the parameter values compatible with the null hypothesis $\left(\Omega_{0}\right)$, and $H_{0}$ is rejected if

$$
\sup \left\{\hat{p}_{N}\left(S_{0} \mid \delta\right): \delta \in \Omega_{0}\right\} \leq \alpha .
$$

If the number of simulated statistics $N$ is chosen so that $\alpha(N+1)$ is an integer, then we have under $H_{0}$ :

$$
\mathrm{P}\left[\sup \left\{\hat{p}_{N}\left(S_{0} \mid \delta\right): \delta \in \Omega_{0}\right\} \leq \alpha\right] \leq \alpha,
$$

which means that we have a test with level $\alpha$; for a proof, see Dufour (2006).

Because of the maximization in the critical region (5.1) the test is called a maximized Monte Carlo (MMC) test. MMC tests provide valid inference under general regularity conditions such as 
almost-unidentified models or time series processes involving unit roots. In particular, even though the moment conditions defining the estimator are derived under the stationarity assumption, this does not question in any way the validity of maximized MC tests, unlike the parametric bootstrap whose distributional theory is based on strong regularity conditions. Only the power of MMC tests may be affected.

A simplified approximate version of the MMC procedure can alleviate its computational load whenever a consistent point or set estimate of $\delta$ is available. To do this, we reformulate the setup in order to allow for an increasing sample size $(T)$.

1. To test the null hypothesis

$$
H_{0}: \bar{\delta} \in \Omega_{0}, \quad \text { with } \Omega_{0} \in \Omega, \quad \Omega_{0} \neq \emptyset,
$$

we use first the observed sample to calculate the relevant statistic denoted by $S_{T 0}$.

2. We consider $C_{T}, T \geq I_{0}$, a sequence of (possibly random) subsets of $\Omega$ instead of $\Omega_{0}$, such that

$$
\lim _{T \rightarrow \infty} \mathrm{P}\left[\bar{\delta} \in C_{T}\right]=1 \text { under } H_{0}
$$

3. For each $\delta \in C_{T}$, we generate $N$ replications of $S: S_{T 1}(\delta), \ldots, S_{T N}(\delta)$, with $T \geq I_{0}$.

4. Using these simulations we compute the corresponding simulated $p$-value function:

$$
\hat{p}_{T N}[y \mid \delta]=\frac{N \hat{G}_{T N}[y \mid \delta]+1}{N+1} .
$$

5. The $p$-value function $\hat{p}_{T N}\left[S_{T 0} \mid \delta\right]$ is maximized with respect to $\delta$ in $C_{T}$, and $H_{0}$ is rejected if

$$
\sup \left\{\hat{p}_{T N}\left(S_{T 0} \mid \delta\right): \delta \in C_{T}\right\} \leq \alpha .
$$

If the number of simulated statistics $N$ is chosen so that $\alpha(N+1)$ is an integer, we have under $H_{0}$ :

$$
\lim _{T \rightarrow \infty} \mathrm{P}\left[\sup \left\{\hat{p}_{T N}\left(S_{T 0} \mid \delta\right): \delta \in C_{T}\right\} \leq \alpha\right] \leq \alpha,
$$

i.e., we control for the level asymptotically.

In practice, it is easy to find a consistent set estimate of $\bar{\delta}$, whenever a consistent point estimate $\hat{\delta}_{T}$ of $\bar{\delta}$ is available (e.g. a GMM estimator or a more efficient estimator such as the SMLE; see Danielsson (1994), Durham (2006, 2007)).

For instance, any set of the form

$$
C_{T}=\left\{\delta \in \Omega:\left\|\hat{\delta}_{T}-\delta\right\|<d\right\}
$$

with $d$ a fixed positive constant independent of $T$, satisfies (5.3). The consistent set estimate MMC (CSEMMC) method is especially useful when the distribution of the test statistic is highly sensitive 
to nuisance parameters. Here, possible discontinuities in the asymptotic distribution are automatically overcome through a numerical maximization over a set that contains the true value of the nuisance parameter with probability one asymptotically (while there is no guarantee for the point estimate to converge sufficiently fast to overcome the discontinuity). It is worth noting that there is no need to maximize the $p$-value function with respect to unidentified parameters under the null hypothesis (which corresponds to $\rho_{12}$ in the two-factor SV framework). Thus, parameters which are unidentified under the null hypothesis can be set to any fixed value and the maximization be performed only over the remaining identified nuisance parameters. When there are several nuisance parameters, one can use simulated annealing, an optimization algorithm which does not require differentiability. Indeed $\hat{G}_{N}\left[S_{0} \mid \delta\right]$ is step-type function which typically has zero derivatives almost everywhere, except on isolated points where it is not differentiable. For an example where this is done on a VAR model involving a large number of nuisance parameters, see Dufour and Jouini (2006).

Finally, if the set $C_{T}$ in (5.4) is reduced to a single point estimate $\hat{\delta}_{T}$, i.e. $C_{T}=\left\{\hat{\delta}_{T}\right\}$, we get a local MC (LMC) test

$$
\hat{p}_{T N}\left(S_{T 0} \mid \hat{\delta}_{T}\right) \leq \alpha,
$$

which can be interpreted as a parametric bootstrap test. Even if $\hat{\delta}_{T}$ is a consistent estimate of $\delta$ (under the null hypothesis), the condition (5.3) is not usually satisfied in this case, so additional assumptions are needed to show that the parametric bootstrap procedure yields an asymptotically valid test. It is computationally less costly but clearly less robust to violations of regularity conditions than the MMC procedure; for further discussion, see Dufour (2006).

\section{Simulation results}

In this section, we present some simulation evidence on the finite-sample properties of the procedures described in the previous sections. In particular, we provide results on the actual level of the Wald, score, LR and $C(\alpha)$-type tests for the three main hypotheses discussed: (1) the hypothesis of non-persistence in volatility (against persistence in volatility); (2) homoskedasticity (against the SV alternative); (3) one-factor SV against a two-factor SV. Three ways of implementing the tests are considered: asymptotic critical values, parametric bootstrap, and MMC. We also present results on power for the three types of hypotheses described above.

The Wald-type statistic [defined in equation (3.5)] is evaluated at the unrestricted method-ofmoments estimator $\hat{\theta}$. The score-type statistic [defined in (3.7)] is evaluated at the restricted estimator $\hat{\theta}_{0}$ which minimizes the criterion $M_{T}^{*}(\theta)$ in (3.1) under the constraint $a_{w}=0$. The $C(\alpha)$-type statistic [defined in (3.11)] is evaluated at the restricted estimator $\tilde{\theta}_{0}$ of $\theta$, where $\tilde{\theta}_{0}$ is obtained by setting $a_{w}=0$ in the analytical expressions of the unrestricted method-of-moments estimator $\hat{\theta}$ in (2.18) - (2.21). Further, the LR-type test statistic $L R(\hat{\Omega}) \equiv \xi_{T}^{C}$ corresponds to the difference between the restricted and the unrestricted optimal values of the objective function, with the restricted objective function evaluated at $\hat{\theta}_{0}$ and $\hat{\Omega} \equiv \Omega(\hat{\theta})$. The weighting matrix $\hat{\Omega}$ is estimated by a Bartlett kernel estimator with the bandwidth varying with the sample size, i.e. $K=\left[0.159 T^{1 / 3}\right]$, where $[\cdot]$ denotes the integer part of the enclosed number; see Newey and West (1994). 
Let $S$ denote the test statistic which alternately takes the form of one of the four test statistics mentioned, and $S_{0}$ the statistic computed from the "pseudo-true" data obtained by simulation under the true data generating process. The critical regions have the following forms:

$$
\mathcal{R}_{a}=\left\{S_{0}>\chi_{\alpha}^{2}(\nu)\right\}
$$

for the asymptotic tests, where $\mathrm{P}\left[\chi^{2}(\nu) \geq \chi_{\alpha}^{2}(\nu)\right]=\alpha$ and $\nu$ is the number of constraints tested,

$$
\mathcal{R}_{B}=\left\{\hat{p}_{N}\left[S_{0} \mid \hat{\delta}^{0}\right] \leq \alpha\right\}
$$

for the bootstrap test, and

$$
\mathcal{R}_{M M C}=\left\{\sup \left\{\hat{p}_{T N}\left(S_{T 0} \mid \delta\right): \delta \in C_{T}\right\} \leq \alpha\right\},
$$

where

$$
\begin{aligned}
\hat{p}_{N}[x \mid \delta] & =\frac{N \hat{G}_{N}[x \mid \delta]+1}{N+1}, \\
\hat{G}_{N}[x ; S(N, \delta)] & =\frac{1}{N} \sum_{i=1}^{N} s\left(S_{i}(\delta)-x\right),
\end{aligned}
$$

for the MMC-based test. $\hat{\delta}^{0}$ is a consistent point restricted estimate of $\delta=\left(c, \theta^{\prime}\right)^{\prime}$,; $\theta$ is the vector of the SV parameters [e.g., $\theta=\left(a_{w}, r_{y}, r_{w}\right)^{\prime}$ for the one-factor SV model, $\theta=$ $\left(a_{w}, r_{y}, r_{w}, a_{\eta}, r_{\eta}, \rho_{12}\right)^{\prime}$ for the two-factor SV model], and $C_{T}$ is a restricted consistent set estimator of $\delta$.

For MMC tests of the non-persistence hypothesis in the single-factor SV model $\left(a_{w}=0\right)$, the set $C_{T}$ over which we maximize the simulated $p$-value is:

$$
C_{T}^{(1)}=\left\{\left(c, r_{y} r_{w}\right):|c-\hat{c}| \leq 0.15,|c| \leq 0.99,\left|r_{y}-\hat{r}_{y}^{(1)}\right| \leq 0.3,\left|r_{w}-\hat{r}_{w}^{(1)}\right| \leq 0.3\right\}
$$

where $\hat{c}$ is the least squares estimates of $c$ [based on fitting the AR(1) model (2.1) with no drift] and $\left(\hat{r}_{y}^{(1)}, \hat{r}_{w}^{(1)}\right)$ are the restricted GMM estimate of $\left(r_{y}, r_{w}\right)$ in the one-factor model [based on minimizing $M_{T}^{*}(\theta)$ subject to the restriction $\left.a_{w}=0\right]$. The bounds of 0.15 for $c$ and 0.3 for the scale parameters approximately correspond to 7 and 2 standard errors, respectively. However, any fixed bound associated with a consistent estimator will lead to an asymptotically valid test provided that the probability of covering the true parameter converges to one as the sample size goes to infinity. For the homoskedasticity hypothesis $\left(a_{w}=r_{w}=0\right)$, the corresponding set is

$$
C_{T}^{(2)}=\left\{\left(c, r_{y}\right):|c-\hat{c}| \leq 0.15,|c| \leq 0.99,\left|r_{y}-\hat{r}_{y}^{(2)}\right| \leq 0.3\right\}
$$

where $\hat{r}_{y}^{(2)}$ is the corresponding restricted GMM estimate of $r_{y}$ [based on minimizing $M_{T}^{*}(\theta)$ subject to the restriction $\left.a_{w}=r_{w}=0\right]$. Finally, for testing the one-factor model against the two-factor 
$\operatorname{model}\left(a_{\eta}=r_{\eta}=0\right), C_{T}$ is

$$
\begin{gathered}
C_{T}^{(3)}=\left\{\left(c, a_{w}, r_{y}, r_{w}\right):|c-\hat{c}| \leq 0.15,|c| \leq 0.99,\left|a_{w}-\hat{a}_{w}^{(3)}\right| \leq 0.15,\left|a_{w}\right| \leq 0.99\right. \\
\left.\left|r_{y}-\hat{r}_{y}^{(3)}\right| \leq 0.3,\left|r_{w}-\hat{r}_{w}^{(3)}\right| \leq 0.3\right\}
\end{gathered}
$$

where $\hat{r}_{y}^{(3)}, \hat{r}_{w}^{(3)}$ and $\hat{a}_{w}^{(3)}$ are restricted moment estimates of the two-factor model [based of the moment equations in (2.28) - (2.33)]. Since the number of nuisance parameters is relatively small, maximization was achieved through a grid search (with points separated by a distance of 0.03 for each coefficient). Note that many other restricted consistent estimates of the relevant nuisance parameters could be used to build the sets $C_{T}$.

The nominal level is $\alpha=0.05$. The number of replications used for Monte Carlo tests is $N=99$, while the rejection frequencies are estimated with $M=1000$ for regular hypothesis tests and $M=500$ for non-regular ones. $T$ is the sample size of the series $y_{t}$ whose data generating process is assumed to be specified as in equations (2.4) - (2.6) for the one-factor SV model and as in equations (2.22) - (2.25) for the two-factor SV model. Calculations were performed with the GAUSS software. The autoregressive parameters $a_{w}$ and $a_{\eta}$ in the autoregressive specifications for the volatility process are restricted to an interval inside $(-1,1)$ to ensure stationarity.

In the power study (Section 6.2), the asymptotic critical points are locally level-corrected, i.e. the critical points are modified to ensure that the rejection frequency under the null hypothesis (for the specific nuisance parameter values considered) is equal to 0.05 ; the corrected critical value is obtained by simulating the test statistic under the null hypothesis with a large number of replications. ${ }^{4}$ Corrected asymptotic critical values are estimated from a simulation with 10000 replications (with 5000 replications) for the regular hypotheses ( for the non-regular hypotheses). Bootstrap tests are level-corrected by decreasing the threshold under which the bootstrap $p$-value must fall to ensure that the bootstrap test rejects with frequency of 0.05 under the null hypothesis; the corrected threshold is estimated from a simulation with 10000 replications (with 5000 replications) for the regular hypotheses (for the non-regular hypotheses).

\subsection{Level}

We will now examine the empirical levels of the tests. The results on testing volatility nonpersistence $\left(H_{0}: a_{w}=0\right)$ are reported in Table 1 (panel A). We can see from this table that all bootstrap procedures exhibit notable over-rejections rates (above the nominal level of 0.05), in small samples (even with a sample of $T=500$ ), while the asymptotic tests under-reject. Indeed, the asymptotic critical values appear to be conservative in this case. The only procedures which do not exhibit over-rejections in the cases considered are the MMC versions of all the tests. As expected from theory, the latter may be conservative.

\footnotetext{
${ }^{4}$ We use the term "locally level-corrected" instead of "size-corrected" because a true size correction would require one to ensure that the probability of rejecting the null hypothesis under all distributions compatible with null hypothesis (i.e., for all values of the nuisance parameters) be less than or equal to the level $\alpha$. Theoretically, a complete size-correction would be the most satisfactory correction to perform for a fair comparison of all the test procedures. However, finding the appropriate size-corrected critical values requires a numerical search that could not be performed in the context of the present experiment.
} 
Table 1. Empirical levels of asymptotic, bootstrap and MMC tests, (nominal size: $\alpha=5 \%$ ).

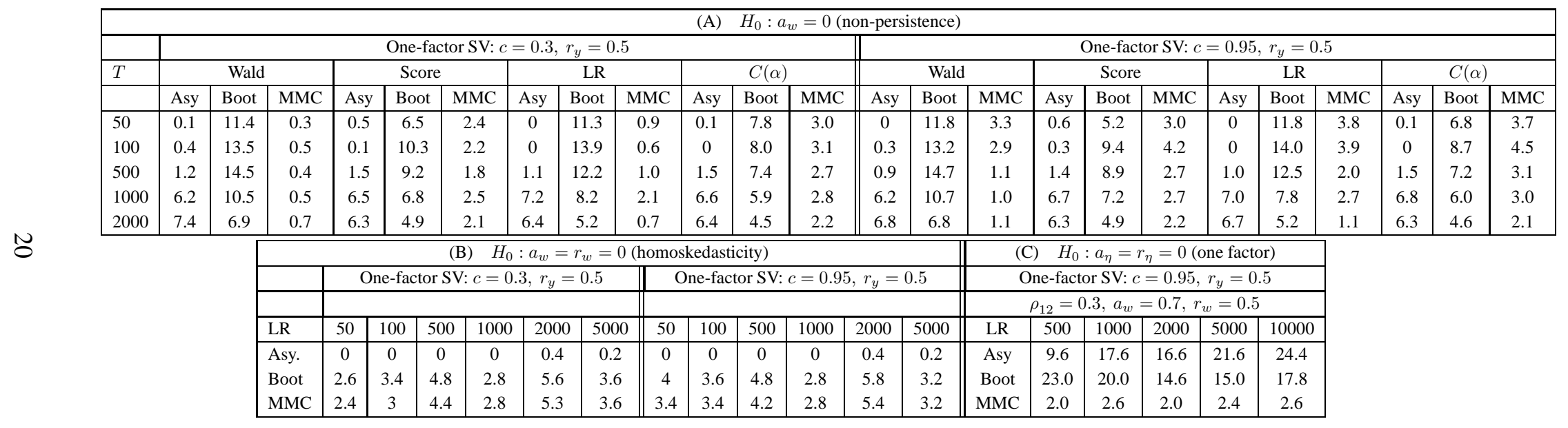

Note - In this table as well as in the other tables, frequencies are reported in percentages. Simulations are computed on 1000 replications for panel (A)while they are computed on 500 replications for panel (B) and (C). 
Table 2. Empirical power of asymptotic, bootstrap and MMC tests.

\begin{tabular}{|c|c|c|c|c|c|c|c|c|c|c|c|c|c|c|c|c|c|c|c|c|c|c|c|c|}
\hline \multicolumn{25}{|c|}{ (A) $\quad H_{1}: a_{w}=0.8$ (persistence) against $H_{0}: a_{w}=0$ (non-persistence) } \\
\hline & \multicolumn{12}{|c|}{ One-factor SV: $c=0.3, r_{y}=0.5$} & \multicolumn{12}{|c|}{ One-factor SV: $c=0.95, r_{y}=0.5$} \\
\hline \multirow[t]{2}{*}{$T$} & \multicolumn{3}{|c|}{ Wald } & \multicolumn{3}{|c|}{ Score } & \multicolumn{3}{|c|}{ LR } & \multicolumn{3}{|c|}{$C(\alpha)$} & \multicolumn{3}{|c|}{ Wald } & \multicolumn{3}{|c|}{ Score } & \multicolumn{3}{|c|}{ LR } & \multicolumn{3}{|c|}{$C(\alpha)$} \\
\hline & Asy & Boot & MMC & Asy & Boot & MMC & Asy & Boot & MMC & Asy & Boot & MMC & Asy & Boot & MMC & Asy & Boot & MMC & Asy & Boot & MMC & Asy & Boot & MMC \\
\hline 50 & 1.4 & 6.4 & 2.2 & 0.4 & 6.5 & 1.2 & 0.1 & 7.9 & 2.6 & 1.1 & 11.4 & 8.3 & 1.9 & 8.0 & 7.6 & 0.8 & 7.5 & 4.7 & 0.2 & 10.5 & 9.4 & 0.4 & 10.6 & 11.7 \\
\hline 100 & 3.6 & 15.5 & 3.3 & 2.1 & 11.7 & 6.4 & 0.5 & 21.5 & 4.0 & 2.8 & 18.3 & 15.7 & 3.6 & 18.0 & 10.4 & 1.3 & 15.5 & 11.4 & 0.8 & 22.5 & 13.5 & 2.3 & 20.6 & 18.7 \\
\hline 500 & 45.9 & 54.5 & 7.9 & 32.5 & 38.2 & 24.7 & 30.6 & 35.1 & 8.6 & 34.9 & 41.2 & 39.5 & 45.4 & 54.4 & 32.0 & 31.5 & 42.6 & 32.7 & 31.9 & 38.0 & 29.5 & 33.4 & 42.5 & 41.4 \\
\hline 1000 & 86.6 & 67.0 & 23.6 & 59.9 & 50.6 & 33.2 & 60.3 & 43.2 & 10.8 & 64.7 & 57.9 & 42.3 & 87.0 & 66.3 & 57.2 & 57.8 & 50.2 & 38.6 & 59.2 & 41.1 & 33.6 & 63.5 & 49.3 & 45.5 \\
\hline 2000 & 99.0 & 90.2 & 54.2 & 81.9 & 80.3 & 59.7 & 84.3 & 83.8 & 39.0 & 86.8 & 82.9 & 70.3 & 99.0 & 91.3 & 83.9 & 82.3 & 81.0 & 68.6 & 84.9 & 84.1 & 67.7 & 87.7 & 83.6 & 74.1 \\
\hline \multicolumn{25}{|c|}{ (B) $H_{1}: a_{w}=0.9$ (persistence) against $H_{0}: a_{w}=0$ (non-persistence) } \\
\hline & \multicolumn{12}{|c|}{ One-factor SV: $c=0.3, r_{y}=0.5$} & \multicolumn{12}{|c|}{ One-factor SV: $c=0.95, r_{y}=0.5$} \\
\hline$T$ & \multicolumn{3}{|c|}{ Wald } & \multicolumn{3}{|c|}{ Score } & \multicolumn{3}{|c|}{ LR } & \multicolumn{3}{|c|}{$C(\alpha)$} & \multicolumn{3}{|c|}{ Wald } & \multicolumn{3}{|c|}{ Score } & \multicolumn{3}{|c|}{ LR } & \multicolumn{3}{|c|}{$C(\alpha)$} \\
\hline & Asy & Boot & MMC & Asy & Boot & MMC & Asy & Boot & MMC & Asy & Boot & MMC & Asy & Boot & MMC & Asy & Boot & MMC & Asy & Boot & MMC & Asy & Boot & MMC \\
\hline 50 & 1.9 & 10.7 & 3.4 & 1.0 & 9.6 & 2.4 & 0.6 & 14.3 & 4.8 & 2.0 & 15.2 & 12.8 & 2.9 & 11.6 & 12.0 & 0.9 & 13.1 & 9.7 & 0.7 & 14.9 & 15.0 & 2.6 & 20.8 & 20.0 \\
\hline 100 & 6.7 & 26.9 & 6.1 & 4.1 & 23.6 & 11.9 & 2.2 & 31.5 & 9.2 & 6.0 & 28.5 & 25.9 & 7.9 & 28.1 & 20.1 & 4.2 & 21.4 & 21.8 & 2.4 & 31.7 & 24.3 & 6.6 & 31.6 & 34.5 \\
\hline 500 & 81.9 & 71.7 & 33.3 & 49.1 & 54.5 & 37.4 & 48.7 & 49.3 & 20.6 & 53.6 & 57.5 & 55.8 & 81.6 & 71.7 & 57.8 & 50.6 & 40.2 & 42.0 & 51.0 & 48.3 & 37.1 & 55.6 & 59.3 & 50.3 \\
\hline 1000 & 96.5 & 84.4 & 63.0 & 58.9 & 54.7 & 38.2 & 59.9 & 53.0 & 21.1 & 66.5 & 62.7 & 56.4 & 97.6 & 83.2 & 87.6 & 59.4 & 57.8 & 46.4 & 61.4 & 54.4 & 38.2 & 70.6 & 64.2 & 58.0 \\
\hline 2000 & 98.9 & 95.4 & 84.2 & 73.0 & 72.3 & 51.6 & 78.9 & 78.4 & 40.1 & 82.4 & 79.2 & 67.0 & 99.1 & 96.0 & 94.1 & 74.4 & 74.0 & 59.5 & 81.0 & 80.3 & 59.5 & 84.6 & 82.1 & 69.6 \\
\hline
\end{tabular}

\begin{tabular}{|c|c|c|c|c|c|c|c|c|c|c|c|c|c|c|c|c|c|c|}
\hline \multirow[t]{3}{*}{ (C) } & \multicolumn{12}{|l|}{$H_{1}$} & \multicolumn{6}{|c|}{ 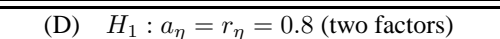 } \\
\hline & & & & & & & & & & & & & \multirow{2}{*}{\multicolumn{6}{|c|}{$\frac{\text { against } H_{0}: a_{\eta}=r_{\eta}=0 \text { (one factor) }}{\text { One-factor SV: } c=0.95, r_{y}=0.5}$}} \\
\hline & \multicolumn{6}{|c|}{ One-factor SV: $c=0.3, r_{y}=0.5$} & \multicolumn{6}{|c|}{ One-factor SV: $c=0.95, r_{y}=0.5$} & & & & & & \\
\hline & & & & & & & & & & & & & \multicolumn{6}{|c|}{$\rho_{12}=0.3, a_{w}=0.7, r_{w}=0.5$} \\
\hline 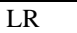 & 50 & 100 & $\overline{500}$ & 1000 & 2000 & 5000 & 50 & 100 & $\overline{500}$ & 1000 & 2000 & 5000 & LR & 500 & 1000 & 2000 & 5000 & 10000 \\
\hline Asy. & 0.2 & 0.6 & 30 & 75.0 & 96.6 & 100 & 0.6 & 1.2 & 30.0 & 75.2 & 96.4 & 100 & Asy & 0.6 & 0.2 & 0.6 & 0.4 & 0.4 \\
\hline Boot & 22.4 & 41.8 & 88.0 & 96.4 & 99.4 & 100 & 25.2 & 40.6 & 86.0 & 96.2 & 98.4 & 100 & Boot & 0.6 & 2.6 & 9.4 & 60.4 & 71.4 \\
\hline MMC & 21.2 & 41.2 & 87.0 & 96.4 & 99.4 & 100 & 22.8 & 38.8 & 85.4 & 95.8 & 99.4 & 100 & MMC & 2.0 & 7.2 & 14.6 & 69.4 & 77.3 \\
\hline
\end{tabular}

Note - All asymptotic tests are locally level-corrected. Bootstrap tests are locally level-corrected when the probability of type I error exceeds 0.05. Locally-level corrected tests are not feasible in practice. Simulations are computed on 1000 replications for panel (A) and (B) while they are computed on 500 replications for panel (C) and (D). All tests statistics are regularized with an identity matrix when numerical instability arises in the simulation experiments leading to (almost) non-invertibility of the covariance matrix. The simulated critical values (obtained on 5000 replications under the true DGP) for the level-corrected asymptotic LR test used in panel (D) are $7.452,13.141,22.848,45.506$ and 77.499 for the sample sizes $T=500,1000,2000,5000$ and 10000, respectively. 
Results on testing homoskedasticity and the one-factor hypothesis appear in Table 1 (panel B and $\mathrm{C}$ ). Because these hypotheses lead to locally singular moment conditions, the score and $C(\alpha)$ type tests are not applicable here, while Wald tests often depend on covariance matrices which are almost singular (generating numerically unstable behavior). So only LR-type tests are considered. We see from the results that asymptotic LR-type tests are very conservative for the homoskedasticity hypothesis but can severely over-reject for the one-factor hypothesis. Indeed, size distortions increase with the sample size, indicating that standard critical values are not asymptotically valid. Bootstrapping appears to correct the situation for the first hypothesis, but leaves notable over-rejection rates in the second case. Of course, one cannot exclude the possibility of larger bootstrap failures for different parameter configurations. Clearly, the two types of non-regular problems studied are qualitatively different from the statistical viewpoint. Again, in all cases studied, the MMC-based tests do not exhibit over-rejection rates.

\subsection{Power}

We will now study the empirical powers of the tests. In Table 2 (panel A and B), we report empirical powers for tests of $H_{0}: a_{w}=0$. We can see from the results that the $C(\alpha)$ and the Wald-type tests (built upon the closed-form moment estimator) have more power than the other tests. Further, the $C(\alpha)$-type test is easy to implement in this context since it does not require any optimization procedure unlike the LR and the score-type tests. Further, although the MMC-based tests may be conservative, their power is in fact quite close to the one of the other tests and even perform better, in some cases, than the level-corrected bootstrap and asymptotic tests in small samples (e.g. for $T=50,100$ in Table 2). In the present situation, MMC-based tests are essentially (almost) equivalent to (infeasible) level-corrected bootstrap tests, which suggest that they may dominate size-corrected bootstrap tests (whose level would be controlled over the whole nuisance-parameter space).

We also examine in Table 2 (panel C) the power of homoskedasticity tests (against one-factor SV). Bootstrap and MMC-based LR-type tests exhibit good power - which increases with the sample size - and are very close to each other. Besides, they over-perform their asymptotic analog for sample sizes less than $T=2000$. Note that the locally level-corrected asymptotic tests are not feasible in practice (because critical values are computed using unknown parameter values under the null hypothesis).

For tests of the one factor hypothesis (panel D of Table 2), the power of LR-type tests - though low in comparison with the other hypotheses studied previously - is sizeable and increases with the sample size. On the other hand, detecting the presence of a second factor in the volatility process requires a sufficiently large sample. Thus, for average sample sizes, the MMC-LR procedure has always more power than the corresponding (infeasible) locally level-corrected bootstrap test, while the corresponding (infeasible) locally level-corrected asymptotic test has no power at all once corrected for size distortions. Thus, the only valid feasible test which guarantees to control for the level - unlike the asymptotic and bootstrap procedures - provides reasonable power. 


\section{Empirical application}

In this section, we test the three null hypotheses studied in the simulation experiments from real data on the Standard and Poor's Composite Price Index (1928-87). We proceed in three steps in order to select the more suitable specification for this specific data set. First, we test for the null of homoskedasticity against an alternative of stochastic volatility. Second, we perform the test of one factor against two factors in the volatility process. And finally, we implement the test of nopersistence in the one-factor volatility process if the one-factor specification is appropriate.

\subsection{Data}

The data have been provided by Georges Tauchen where the efficient method of moments (EMM) have been used by Gallant et al. (1997) to fit a SV model. The data to which we fit the stochastic volatility models is a time series comprised of 16,127 daily observations, $\left\{\tilde{y}_{t}\right\}_{t=1}^{16,127}$, on adjusted movements of the Standard and poor's Composite Price Index, 1928-87. The raw series is the Standard and Poor's Composite Price Index (SP), 1928-87 (daily). The raw series is converted to growth rates by the transformation $100\left[\log \left(S P_{t}\right)-\log \left(S P_{t-1}\right)\right]$, and then adjusted for systematic calendar effects, that is, systematic shifts in location and scale due to different trading patterns across days of the week, holidays, and year-end tax trading.

\subsection{Results}

The unrestricted estimated value of $(c, \theta)$ for the one-factor model obtained from the data is:

$$
\begin{aligned}
& {\left[\hat{c}, \hat{a}_{w}, \hat{r}_{y}, \hat{r}_{w}\right]=\left[\begin{array}{llll}
0.129, & 0.926, & 0.829, & 0.427
\end{array}\right]} \\
& \begin{array}{llll}
(0.007) & (8.10) & (1.91) & (8.13)
\end{array}
\end{aligned}
$$

where standard errors are given in parentheses; the latter are computed from the square root of $\left(\hat{J}^{\prime} \hat{I}^{-1} \hat{J}\right)^{-1}$. We may conjecture that there is some persistence in the data during the period 192887 what is statistically checked by performing the tests below. The restricted estimated values of $(c, \theta)$ from the data are:

$$
\left.\left[\hat{c}, \hat{a}_{w}^{0}, \hat{r}_{y}^{0}, \hat{r}_{w}^{0}\right]=\begin{array}{cccc}
{[0.129,} & 0, & 0.785, & 1.152
\end{array}\right]
$$

and the consistent restricted estimator derived from the closed-form expression of the unrestricted moment estimator is equal to

$$
\left.\left[\hat{c}, \tilde{a}_{w}^{0}, \tilde{r}_{y}^{0}, \tilde{r}_{w}^{0}\right]=\begin{array}{llll}
{[0.129,} & 0, & 0.829, & 1.133
\end{array}\right] .
$$

Note the large discrepancy between the unrestricted and restricted estimates of $r_{w}$ where the restricted estimates may not be consistent if the null hypothesis $H_{0}: a_{w}=0$ is false.

In Table 3, we report tests based on the whole sample (16,127 daily observations on the S\&P 500), which covers the market crashes of the Black Thursday of October 1929 and of October 1987, 
the Cuban Missile Crisis (October 1962) and the Arab Oil Embargo (October 1973). We then repeat the tests on three sub-samples: 1928 - 1949, 1950 - 1969 and $1970-1987$.

We can see from the results in panel A of Table 3, that the three versions (asymptotic, bootstrap and MMC) of the LR test do reject the null hypothesis of homoskedasticity in favor of a stochastic volatility specification for the volatility process of the S\&P 500 index, except for the third sub-period 1970-1987 but with $p$-values however very close to 0.05 ( $p$-value $=0.07)$.

More recently, Chernov et al. (2003) and Durham (2006, 2007) provide evidence that standard single-factor SV models have some difficulties to model the shape of the conditional distribution of financial returns. In particular, Chernov et al. (2003) show that two-factor SV models better accommodate richer dynamics such as the tail behavior of (conditional) return distributions and possibly capture some rapid moves in the dynamics of volatility during extreme market conditions. The first factor may act as a long-memory component, while the second factor is expected to model tail behavior. To check for that, we test the null of one factor against two factors $\left(H_{0}: a_{\eta}=r_{\eta}=0\right)$ in panel B of Table 3. All versions (asymptotic, bootstrap and MMC) of the LR test do not indicate that a two-factor specification is needed for the S\&P 500 index volatility. Consequently, we chose a one-factor specification for modelling the S\&P 500 index volatility.

We will now study in greater detail the volatility parameter in the one-factor SV model by testing the null hypothesis of no-persistence in the volatility process (panel $\mathrm{C}$ in Table 3). All tests, asymptotic, bootstrap and MMC, reject the null hypothesis of no-persistence in the volatility for all the periods considered. Indeed, it is well known in the financial literature that financial returns display serial dependence in volatility.

To summarize, the results presented here indicate that a one-factor model with strong volatility persistence may be appropriate for the S\&P 500 index data studied here.

\section{Conclusion}

In this paper, we have provided finite-sample procedures for testing hypotheses on the parameters of SV models, allowing for the possible presence of non-regular testing problems (underidentification, singularity issues) that can lead to non-standard asymptotic distributional theory. Besides usual linear restrictions on SV coefficients, the problems studied include testing homoskedasticity against a SV alternative and testing the one-factor SV against two-factor SV, which raises singularity and identification difficulties. In addition to the three standard tests, we proposed to use $C(\alpha)$-type tests which are relatively easy to apply and displays good size and power properties (when applicable).

In order to deal with the potential unreliability of asymptotic critical values and bootstrapping, especially in cases where standard regularity conditions fail, we showed that the MMC test approach provides a transparent way of dealing with such difficulties, yielding both exact or asymptotically valid tests without the need to establish a specific distributional theory. In some cases (underidentification case), the MMC method is the only one that yields provably valid tests. Further, in simulations, we observed that the MMC method can indeed be implemented to produce valid inference on SV models, works well from the viewpoint of controlling test levels, and most of the time, does not entail a considerable power loss with respect to alternative (usually infeasible) level-corrected asymptotic or bootstrap approaches. 
Table 3. Empirical application on S\&P500 index.

\begin{tabular}{|c|c|c|c|c|c|c|c|c|c|c|c|c|c|c|}
\hline & \multicolumn{7}{|c|}{ (A) $\quad$ Test of homoskedasticity $\quad H_{0}: a_{w}=r_{w}=0$} & \multicolumn{7}{|c|}{ (B) Test of one factor against two SV factors $\quad H_{0}: a_{w}=r_{w}=0$} \\
\hline & Asymptotic tests & \multicolumn{3}{|c|}{ Bootstrap tests } & \multicolumn{3}{|c|}{ MMC tests } & Asymptotic tests & \multicolumn{3}{|c|}{ Bootstrap tests } & \multicolumn{3}{|c|}{ MMC tests } \\
\hline & $S_{0}$ & $N=19$ & $N=99$ & $N=999$ & $N=19$ & $N=99$ & $N=999$ & $S_{0}$ & $N=19$ & $N=99$ & $N=999$ & $N=19$ & $N=99$ & $N=999$ \\
\hline & \multicolumn{7}{|c|}{ Sample 1928-1987, $T=16127$} & \multicolumn{7}{|c|}{ Sample 1928-1987, $T=16127$} \\
\hline \multirow[t]{2}{*}{ LR } & 9.71 & 0.05 & 0.01 & 0.001 & 0.05 & 0.01 & 0.001 & 4.149 & 0.35 & 0.33 & 0.329 & 0.70 & 0.70 & 0.681 \\
\hline & \multicolumn{7}{|c|}{ Sample 1928-1949, $T=6491$} & \multicolumn{7}{|c|}{ Sample 1928-1949, $T=6491$} \\
\hline \multirow[t]{2}{*}{ LR } & 42.58 & 0.05 & 0.01 & 0.001 & 0.05 & 0.01 & 0.001 & 0.435 & 0.85 & 0.84 & 0.813 & 0.85 & 0.84 & 0.813 \\
\hline & \multicolumn{7}{|c|}{ Sample $1950-1969, T=5087$} & \multicolumn{7}{|c|}{ Sample $1950-1969, T=5087$} \\
\hline \multirow[t]{2}{*}{ LR } & 6.28 & 0.05 & 0.01 & 0.003 & 0.5 & 0.02 & 0.003 & 2.700 & 0.30 & 0.21 & 0.153 & 0.35 & 0.24 & 0.222 \\
\hline & \multicolumn{7}{|c|}{ Sample 1970-1987, $T=4549$} & \multicolumn{7}{|c|}{ Sample 1970-1987, $T=4549$} \\
\hline \multirow[t]{5}{*}{ LR } & 2.09 & 0.10 & 0.07 & 0.077 & 0.10 & 0.07 & 0.078 & 1.141 & 0.40 & 0.28 & 0.205 & 0.80 & 0.77 & 0.747 \\
\hline & \multicolumn{7}{|c|}{ (C) Test of no-persistence $\quad H_{0}: a_{w}=0$} & \multicolumn{7}{|c|}{ (C) Test of no-persistence $\quad H_{0}: a_{w}=0$} \\
\hline & Asymptotic tests & \multicolumn{3}{|c|}{ Bootstrap tests } & \multicolumn{3}{|c|}{ MMC tests } & Asymptotic tests & \multicolumn{3}{|c|}{ Bootstrap tests } & \multicolumn{3}{|c|}{ MMC tests } \\
\hline & $S_{0}$ & $N=19$ & $N=99$ & $N=999$ & $N=19$ & $N=99$ & $N=999$ & $S_{0}$ & $N=19$ & $N=99$ & $N=999$ & $N=19$ & $N=99$ & $N=999$ \\
\hline & \multicolumn{7}{|c|}{ Sample $1928-1987, T=16127$} & \multicolumn{7}{|c|}{ Sample $1950-1969, T=5087$} \\
\hline Wald & 210.85 & 0.05 & 0.01 & 0.001 & 0.05 & 0.01 & 0.001 & 93.01 & 0.05 & 0.01 & 0.001 & 0.05 & 0.01 & 0.001 \\
\hline Score & 1039.04 & 0.05 & 0.01 & 0.001 & 0.05 & 0.01 & 0.001 & 607.92 & 0.05 & 0.01 & 0.001 & 0.05 & 0.01 & 0.001 \\
\hline 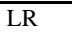 & 25.49 & 0.05 & 0.01 & 0.001 & 0.05 & 0.01 & 0.001 & 11.95 & 0.05 & 0.01 & 0.001 & 0.05 & 0.01 & 0.001 \\
\hline$C(\alpha)$ & 854.55 & 0.05 & 0.01 & 0.001 & 0.05 & 0.01 & 0.001 & 304.66 & 0.05 & 0.01 & 0.001 & 0.05 & 0.01 & 0.001 \\
\hline & \multicolumn{7}{|c|}{ Sample 1928-1949, $T=6491$} & \multicolumn{7}{|c|}{ Sample 1970-1987, $T=4549$} \\
\hline Wald & 112.95 & 0.05 & 0.01 & 0.001 & 0.05 & 0.01 & 0.001 & 30.50 & 0.05 & 0.01 & 0.001 & 0.10 & 0.03 & 0.027 \\
\hline Score & 269.72 & 0.05 & 0.01 & 0.001 & 0.05 & 0.01 & 0.001 & 391.87 & 0.05 & 0.01 & 0.001 & 0.05 & 0.01 & 0.001 \\
\hline LR & 52.73 & 0.05 & 0.01 & 0.001 & 0.05 & 0.01 & 0.001 & 40.90 & 0.05 & 0.01 & 0.001 & 0.05 & 0.01 & 0.001 \\
\hline$C(\alpha)$ & 185.47 & 0.05 & 0.01 & 0.001 & 0.05 & 0.01 & 0.001 & 165.03 & 0.05 & 0.01 & 0.001 & 0.05 & 0.01 & 0.001 \\
\hline
\end{tabular}

A varying bandwidth may entail some power loss in large samples, therefore we set the bandwidth to $K=1$ to maximize power in the empirical application for all tests. 
It is worth noting that Monte Carlo tests do not hinge on the method-of-moment estimator used here. These can be applied as soon as a consistent estimator is available (e.g. GMM, SMM, EMM or indirect inference). The only restriction is that the test statistic can be simulated, without the need to study its asymptotic distribution nor even to establish its existence. However, using estimators with better finite-sample properties such as the SMLE [see Danielsson (1994), Durham (2006, 2007)], might improve the performance of the tests, but this issue goes beyond the scope of the present paper.

These testing procedures can easily be extended to accommodate richer dynamics such as fattailed and/or correlated errors [see Jacquier, Polson and Rossi (2004)], or multivariate stochastic volatility structures [see Chib, Nardari and Shephard (2006)]. 


\section{A. Appendix: Proofs}

Proof of Proposition 2.4 If $U \sim \mathrm{N}(0,1)$ then $E\left(U^{2 p+1}\right)=0, \forall p \in \mathbb{N}$ and $E\left(U^{2 p}\right)=$ $(2 p) ! /\left[2^{p} p !\right] \forall p \in \mathbb{N}$. Hence:

$$
\begin{aligned}
\mathrm{E}\left(u_{t}^{k}\right) & =r_{y}^{k} \mathrm{E}\left(z_{t}^{k}\right) \mathrm{E} \exp \left[k\left(w_{t} / 2+\eta_{t} / 2\right)\right] \\
& =r_{y}^{k} \frac{k !}{2^{(k / 2)}(k / 2) !} \exp \left[\frac{k^{2}}{8}\left(\operatorname{Var}\left(w_{t}\right)+\operatorname{Var}\left(\eta_{t}\right)+2 \operatorname{Cov}\left(w_{t}, \eta_{t}\right)\right)\right] \\
& =r_{y}^{k} \frac{k !}{2^{(k / 2)}(k / 2) !} \exp \left[\frac{k^{2}}{8} r_{w}^{2} /\left(1-a_{w}^{2}\right)+\frac{k^{2}}{8} r_{\eta}^{2} /\left(1-a_{\eta}^{2}\right)+\frac{k^{2}}{4} \frac{r_{w} r_{\eta} \rho_{12}}{1-a_{w} a_{\eta}}\right]
\end{aligned}
$$

where the second equality uses the definition of the Gaussian Laplace transform of $w_{t} \sim$ $\mathrm{N}\left[0, r_{w}^{2} /\left(1-a_{w}^{2}\right)\right]$ (of $\eta$ respectively) and of the moments of $z_{t}$. Further, using

$$
\begin{gathered}
\mathrm{E}\left(w_{t}\right)=0, \quad \operatorname{Var}\left(w_{t}\right)=r_{w}^{2} /\left(1-a_{w}^{2}\right) \\
\mathrm{E}\left(\eta_{t}\right)=0, \operatorname{Var}\left(\eta_{t}\right)=r_{\eta}^{2} /\left(1-a_{\eta}^{2}\right), \\
\operatorname{Cov}\left(w_{t}, w_{t+l}\right)=a_{w}^{|l|} r_{w}^{2} /\left(1-a_{w}^{2}\right) \operatorname{Cov}\left(\eta_{t}, \eta_{t+l}\right)=a_{\eta}^{|l|} r_{\eta}^{2} /\left(1-a_{\eta}^{2}\right), \\
\operatorname{Cov}\left(w_{t}, \eta_{t}\right)=\frac{r_{w} r_{\eta} \rho_{12}}{1-a_{w} a_{\eta}}, \\
\operatorname{Cov}\left(w_{t}, \eta_{t+l}\right)=a_{\eta}^{|l|} \operatorname{Cov}\left(w_{t}, \eta_{t}\right), \operatorname{Cov}\left(w_{t+l}, \eta_{t}\right)=a_{w}^{|l|} \operatorname{Cov}\left(w_{t}, \eta_{t}\right)
\end{gathered}
$$

we obtain the cross-moments:

$$
\begin{aligned}
& \mathrm{E}\left[u_{t}^{j} u_{t+l}^{k}\right]=\mathrm{E}\left\{r_{y}^{j+k} z_{t}^{j} z_{t+l}^{k} \exp \left[j\left(\frac{w_{t}}{2}+\frac{\eta_{t}}{2}\right)+k\left(\frac{w_{t+l}}{2}+\frac{\eta_{t+l}}{2}\right)\right]\right\} \\
& =r_{y}^{j+k} \mathrm{E}\left(z_{t}^{j}\right) \mathrm{E}\left(z_{t+l}^{k}\right) \mathrm{E}\left\{\exp \left[j\left(\frac{w_{t}}{2}+\frac{\eta_{t}}{2}\right)+k\left(\frac{w_{t+l}}{2}+\frac{\eta_{t+l}}{2}\right)\right]\right\} \\
& =r_{y}^{j+k} \frac{j !}{2^{(j / 2)}(j / 2) !} \frac{k !}{2^{(k / 2)}(k / 2) !} \exp \left\{\frac{1}{2} \operatorname{Var}\left[\frac{j}{2}\left(w_{t}+\eta_{t}\right)+\frac{k}{2}\left(w_{t+l}+\eta_{t+l}\right)\right]\right\} \\
& =r_{y}^{j+k} \frac{j !}{2^{(j / 2)}(j / 2) !} \frac{k !}{2^{(k / 2)}(k / 2) !} \exp \left\{\frac { 1 } { 2 } \left[\frac{j^{2}}{4} \operatorname{Var}\left(w_{t}\right)+\frac{j^{2}}{4} \operatorname{Var}\left(\eta_{t}\right)+\frac{k^{2}}{4} \operatorname{Var}\left(w_{t+l}\right)\right.\right. \\
& +\frac{k^{2}}{4} \operatorname{Var}\left(\eta_{t+l}\right)+\frac{2 j^{2}}{4} \operatorname{Cov}\left(w_{t}, \eta_{t}\right)+\frac{2 j k}{4} \operatorname{Cov}\left(w_{t}, w_{t+l}\right) \\
& +\frac{2 j k}{4} \operatorname{Cov}\left(w_{t}, \eta_{t+l}\right)+\frac{2 j k}{4} \operatorname{Cov}\left(\eta_{t}, w_{t+l}\right)+\frac{2 j k}{4} \operatorname{Cov}\left(\eta_{t}, \eta_{t+l}\right) \\
& \left.\left.+\frac{2 k^{2}}{4} \operatorname{Cov}\left(w_{t+l}, \eta_{t+l}\right)\right]\right\} \\
& =r_{y}^{j+k} \frac{j !}{2^{(j / 2)}(j / 2) !} \frac{k !}{2^{(k / 2)}(k / 2) !} \exp \left[\frac{r_{w}^{2}}{8\left(1-a_{w}^{2}\right)}\left(j^{2}+k^{2}+2 j k a_{w}^{|l|}\right)\right. \\
& +\frac{r_{\eta}^{2}}{8\left(1-a_{\eta}^{2}\right)}\left(j^{2}+k^{2}+2 j k a_{\eta}^{|l|}\right)
\end{aligned}
$$




$$
\left.+\frac{1}{8}\left[2 j^{2}+2 k^{2}+2 j k a_{\eta}^{|l|}+2 j k a_{w}^{|l|}\right] \frac{r_{w} r_{\eta} \rho_{12}}{1-a_{w} a_{\eta}}\right] .
$$

\section{B. Appendix: Analytical moment derivatives for one-factor SV}

The analytical expressions of the derivatives of the moment conditions are given by:

$$
\begin{gathered}
\frac{\partial \mu_{2}}{\partial a_{w}}=\frac{a_{w}}{\left(1-a_{w}^{2}\right)^{2}} r_{w}^{2} r_{y}^{2} \exp \left[\frac{r_{w}^{2}}{2\left(1-a_{w}^{2}\right)}\right], \quad \frac{\partial \mu_{2}}{\partial r_{w}}=\frac{r_{w}}{\left(1-a_{w}^{2}\right)} r_{y}^{2} \exp \left[\frac{r_{w}^{2}}{2\left(1-a_{w}^{2}\right)}\right], \\
\frac{\partial \mu_{2}}{\partial r_{y}}=2 r_{y} \exp \left[\frac{r_{w}^{2}}{2\left(1-a_{w}^{2}\right)}\right], \quad \frac{\partial \mu_{4}}{\partial a_{w}}=12 \frac{a_{w}}{\left(1-a_{w}^{2}\right)^{2}} r_{w}^{2} r_{y}^{4} \exp \left[\frac{2 r_{w}^{2}}{\left(1-a_{w}^{2}\right)}\right], \\
\frac{\partial \mu_{4}}{\partial r_{w}}=12 \frac{r_{w}}{\left(1-a_{w}^{2}\right)} r_{y}^{4} \exp \left[\frac{2 r_{w}^{2}}{\left(1-a_{w}^{2}\right)}\right], \quad \frac{\partial \mu_{4}}{\partial r_{y}}=12 r_{y}^{3} \exp \left[\frac{2 r_{w}^{2}}{\left(1-a_{w}^{2}\right)}\right], \\
\frac{\partial \mu_{2,2}}{\partial a_{w}}=\frac{r_{w}^{2}}{\left(1-a_{w}\right)^{2}} r_{y}^{4} \exp \left[\frac{r_{w}^{2}}{\left(1-a_{w}\right)}\right], \quad \frac{\partial \mu_{2,2}}{\partial r_{w}}=\frac{2 r_{w}}{1-a_{w}} r_{y}^{4} \exp \left[\frac{r_{w}^{2}}{\left(1-a_{w}\right)}\right], \\
\frac{\partial \mu_{2,2}}{\partial r_{y}}=4 r_{y}^{3} \exp \left[\frac{r_{w}^{2}}{\left(1-a_{w}\right)}\right] .
\end{gathered}
$$

All these derivatives evaluated at $a_{w}=0, r_{w}=0$ gives the results stated in equation (4.1).

\section{Appendix: Analytical moment derivatives for two-factor SV}

The analytical expressions of the derivatives of the moment conditions for the two-factor SV model are given by:

$$
\begin{gathered}
\frac{\partial \mu_{2}\left(\theta_{2}\right)}{\partial a_{\eta}}=\left[\frac{a_{\eta} r_{\eta}^{2}}{\left(1-a_{\eta}^{2}\right)^{2}}+\frac{a_{w} r_{w} r_{\eta} \rho_{12}}{\left(1-a_{w} a_{\eta}\right)^{2}}\right] r_{y}^{2} \exp \left(\frac{1}{2} \frac{r_{w}^{2}}{1-a_{w}^{2}}+\frac{1}{2} \frac{r_{\eta}^{2}}{1-a_{\eta}^{2}}+\frac{r_{w} r_{\eta} \rho_{12}}{1-a_{w} a_{\eta}}\right), \\
\frac{\partial \mu_{4}\left(\theta_{2}\right)}{\partial a_{\eta}}=\left[\frac{4 a_{\eta} r_{\eta}^{2}}{\left(1-a_{\eta}^{2}\right)^{2}}+\frac{4 a_{w} r_{w} r_{\eta} \rho_{12}}{\left(1-a_{w} a_{\eta}\right)^{2}}\right] 3 r_{y}^{4} \exp \left(\frac{2 r_{w}^{2}}{1-a_{w}^{2}}+\frac{2 r_{\eta}^{2}}{1-a_{\eta}^{2}}+\frac{4 r_{w} r_{\eta} \rho_{12}}{1-a_{w} a_{\eta}}\right), \\
\frac{\partial \mu_{6}\left(\theta_{2}\right)}{\partial a_{\eta}}=\left[\frac{9 a_{\eta} r_{\eta}^{2}}{\left(1-a_{\eta}^{2}\right)^{2}}+\frac{9 a_{w} r_{w} r_{\eta} \rho_{12}}{\left(1-a_{w} a_{\eta}\right)^{2}}\right] 15 r_{y}^{6} \exp \left(\frac{9}{2} \frac{r_{w}^{2}}{1-a_{w}^{2}}+\frac{9}{2} \frac{r_{\eta}^{2}}{1-a_{\eta}^{2}}+\frac{9 r_{w} r_{\eta} \rho_{12}}{1-a_{w} a_{\eta}}\right), \\
\frac{\partial \mu_{2,2}\left(\theta_{2}\right)}{\partial a_{\eta}}=(1 / 2) r_{y}^{4} \exp \left(\frac{\sigma^{2}}{2}\right) \frac{\partial \sigma^{2}}{\partial a_{\eta}}, \quad \frac{\partial \mu_{4,4}\left(\theta_{2}\right)}{\partial a_{\eta}}=18 r_{y}^{8} \exp \left(2 \sigma^{2}\right) \frac{\partial \sigma^{2}}{\partial a_{\eta}}, \\
\frac{\partial \mu_{6,6}\left(\theta_{2}\right)}{\partial a_{\eta}}=(9 / 2) r_{y}^{12} 225 \exp \left(\frac{9 \sigma^{2}}{2}\right) \frac{\partial \sigma^{2}}{\partial a_{\eta}},
\end{gathered}
$$


where

$$
\frac{\partial \sigma^{2}}{\partial a_{\eta}}=\frac{4 a_{\eta} r_{\eta}^{2}}{\left(1-a_{\eta}^{2}\right)^{2}}+\frac{4 a_{w} r_{w} r_{\eta} \rho_{12}}{\left(1-a_{w} a_{\eta}\right)^{2}}+\frac{2 a_{w}^{2} r_{w} r_{\eta} \rho_{12}}{\left(1-a_{w} a_{\eta}\right)^{2}}+\frac{2 r_{w} r_{\eta} \rho_{12}}{\left(1-a_{w} a_{\eta}\right)^{2}}+\frac{2 a_{\eta}^{2} r_{\eta}^{2}+2 r_{\eta}^{2}}{\left(1-a_{\eta}^{2}\right)^{2}}
$$

All these partial derivatives w.r.t. $a_{\eta}$ take the value zero when evaluated at $a_{\eta}=r_{\eta}=0$. This entails that the Jacobian of the moment conditions does not have full-column rank without the need to compute the other partial derivatives. 


\section{References}

Andersen, T. G., Chung, H.-J. and Sørensen, B. E. (1999), 'Efficient method of moments estimation of a stochastic volatility model: A Monte Carlo study', Journal of Econometrics 91, 61-87.

Andersen, T. G. and Sørensen, B. E. (1996), 'GMM estimation of a stochastic volatility model: A Monte Carlo study', Journal of Business and Economic Statistics 14(3), 328-352.

Andrews, D. W. K. (1987), 'Asymptotic results for generalized Wald tests', Econometric Theory 3, 348-358.

Andrews, D. W. K. (2001), 'Testing when a parameter is on the boundary of the maintained hypothesis', Econometrica 69, 683-733.

Andrews, D. W. K. and Ploberger, W. (1995), 'Admissibility of the likelihood ratio test when a parameter is present only under the alternative', The Annals of Statistics 23, 1383-1414.

Barnard, G. A. (1963), 'Comment on 'The spectral analysis of point processes' by M. S. Bartlett', Journal of the Royal Statistical Society, Series B 25, 294.

Bera, A. K. and Ra, S. (1995), 'A test for the presence of conditional heteroscedasticity within ARCH-M framework', Econometric Reviews 14, 473-485.

Bera, A., Ra, S. and Sarkar, N. (1998), Hypothesis Testing for some Nonregular Cases in Econometrics, in Econometrics: Theory and Practice S. Chakravarty, D. Conddo and R. Mukherjee. Allied Publishers, Mumbai, India, pp. 319-351.

Carnero, M. A., Peña, D. and Ruiz, E. (2004), 'Persistence and kurtosis in GARCH and stochastic volatility models', Journal of Financial Econometrics 2(2), 319-342.

Chernov, M., Gallant, A. R., Ghysels, E. and Tauchen, G. (2003), 'Alternative models for stock price dynamics', Journal of Econometrics 116, 225-257.

Chib, S., Nardari, F. and Shephard, N. (2002), 'Markov chain Monte Carlo methods for stochastic volatility models', Journal of Econometrics 108, 281-316.

Chib, S., Nardari, F. and Shephard, N. (2006), 'Analysis of high dimensional multivariate stochastic volatility models', Journal of Econometrics 134(2), 341-371.

Dagenais, M. G. and Dufour, J.-M. (1991), 'Invariance, nonlinear models and asymptotic tests', Econometrica 59, 1601-1615.

Danielsson, J. (1994), 'Stochastic volatility in asset prices: Estimation with simulated maximum likelihood', Journal of Econometrics 61, 375-400.

Danielsson, J. and Richard, J.-F. (1993), 'Accelerated gaussian importance sampler with application to dynamic latent variable models', Journal of Applied Econometrics 8, S153-S173. 
Davidson, R. and MacKinnon, J. G. (1993), Estimation and Inference in Econometrics, Oxford University Press, New York.

Davies, R. B. (1977), 'Hypothesis testing when a nuisance parameter is present only under the alternative', Biometrika 64, 247-254.

Davies, R. B. (1987), 'Hypothesis testing when a nuisance parameter is present only under the alternative', Biometrika 74, 33-43.

Dufour, J.-M. (1989), 'Nonlinear hypotheses, inequality restrictions, and non-nested hypotheses: Exact simultaneous tests in linear regressions', Econometrica 57, 335-355.

Dufour, J.-M. (1997), 'Some impossibility theorems in econometrics, with applications to structural and dynamic models', Econometrica 65, 1365-1389.

Dufour, J.-M. (2003), 'Identification, weak instruments and statistical inference in econometrics', Canadian Journal of Economics 36(4), 767-808.

Dufour, J.-M. (2006), 'Monte Carlo tests with nuisance parameters: A general approach to finitesample inference and nonstandard asymptotics in econometrics', Journal of Econometrics 133(2), 443-477.

Dufour, J.-M. and Jouini, T. (2006), 'Finite-sample simulation-based tests in VAR models with applications to Granger causality testing', Journal of Econometrics 135(1-2), 229-254.

Dufour, J.-M. and Khalaf, L. (2001), Monte Carlo test methods in econometrics, in B. Baltagi, ed., 'Companion to Theoretical Econometrics', Blackwell Companions to Contemporary Economics, Basil Blackwell, Oxford, U.K., chapter 23, pp. 494-519.

Dufour, J.-M. and Khalaf, L. (2002), 'Simulation based finite and large sample tests in multivariate regressions', Journal of Econometrics 111(2), 303-322.

Dufour, J.-M., Khalaf, L., Bernard, J.-T. and Genest, I. (2004), 'Simulation-based finite-sample tests for heteroskedasticity and ARCH effects', Journal of Econometrics 122(2), 317-347.

Dufour, J.-M. and Trognon, A. (2001), Invariant tests based on $M$-estimators, estimating functions, and the generalized method of moments, Technical report, C.R.D.E., Université de Montréal. 23 pages.

Dufour, J.-M. and Valéry, P. (2006), On a simple two-stage closed-form estimator for a stochastic volatility in a general linear regression, in T. B. Fomby and D. Terrell, eds, 'Advances in Econometrics, Volume 20: Econometric Analysis of Economic and Financial Time Series, in Honor of Clive Granger and Robert Engle, Part A', Elsevier Science, Oxford, U.K., pp. 259288.

Durham, G. B. (2006), 'Monte Carlo methods for estimating, smoothing, and filtering one- and two-factor stochastic volatility models', Journal of Econometrics 133, 273-305. 
Durham, G. B. (2007), 'SV mixture models with application to S\&P 500 index returns', Journal of Financial Economics 85(3), 822-856.

Dwass, M. (1957), 'Modified randomization tests for nonparametric hypotheses', Annals of Mathematical Statistics 28, 181-187.

Engle, R. F. (1982), 'Autoregressive conditional heteroscedasticity with estimates of the variance of United Kingdom inflation’, Econometrica 50(4), 987-1008.

Gallant, A. R., Hsieh, D. and Tauchen, G. (1997), 'Estimation of stochastic volatility models with diagnostics', Journal of Econometrics 81(1), 159-192.

Hansen, B. E. (1996), 'Inference when a nuisance parameter is not identified under the null hypothesis', Econometrica 64, 413-430.

Jacquier, E., Polson, N. G. and Rossi, P. (2004), 'Bayesian analysis of stochastic volatility models with fat-tails and correlated errors', Journal of Econometrics 122, 185-212.

Jacquier, E., Polson, N. and Rossi, P. (1994), 'Bayesian analysis of stochastic volatility models (with discussion)', Journal of Economics and Business Statistics 12, 371-417.

Lütkepohl, H. and Burda, M. M. (1997), 'Modified Wald tests under nonregular conditions', Journal of Econometrics 78, 315-332.

Monfardini, C. (1998), 'Estimating stochastic volatility models through indirect inference', The Econometrics Journal 1(1), C113-C128.

Newey, W. K. and McFadden, D. (1994), Large sample estimation and hypothesis testing, in R. F. Engle and D. L. McFadden, eds, 'Handbook of Econometrics, Volume 4', North-Holland, Amsterdam, chapter 36, pp. 2111-2245.

Newey, W. K. and West, K. D. (1994), 'Automatic lag selection in covariance estimation', Review of Economic Studies 61, 631-654.

Neyman, J. (1959), Optimal asymptotic tests of composite statistical hypotheses, in U. Grenander, ed., 'Probability and Statistics, the Harald Cramér Volume', Almqvist and Wiksell, Uppsala, Sweden, pp. 213-234.

Pierce, A. P. (1982), 'The asymptotic effect of substituting estimators for parameters in certain types of statistics', The Annals of Statistics 10(2), 475-478.

Ruiz, E. (1994), 'Quasi-maximum likelihood estimation of stochastic variance models', Journal of Econometrics 63, 284-306.

Stock, J. H., Wright, J. H. and Yogo, M. (2002), 'A survey of weak instruments and weak identification in generalized method of moments', Journal of Business and Economic Statistics 20(4), 518-529.

Taylor, S. J. (1994), 'Modelling stochastic volatility', Mathematical Finance 4, 183-204. 